\title{
Phase matching, strong frequency doubling and outstanding laser-induced damage threshold in the biaxial, quaternary diamond-like semiconductor $\mathrm{Li}_{4} \mathrm{CdSn}_{2} \mathrm{~S}_{7}$.
}

\author{
Jian-Han Zhang, ${ }^{a}$ Stanislav S. Stoyko, ${ }^{b}$ Andrew J. Craig, ${ }^{b}$ Pedro Grima, ${ }^{c, d}$ Joshua W. Kotchey, ${ }^{b}$ \\ Joon I. Jang ${ }^{e^{*}}$ and Jennifer A. Aitken ${ }^{b^{*}}$ \\ ${ }^{a}$ School of Resources and Chemical Engineering, Sanming University, Sanming, 365004, P. R. China \\ ${ }^{b}$ Department of Chemistry and Biochemistry, Duquesne University, Pittsburgh, PA 15282, USA \\ 'Centro de Estudios de Semiconductores, Departamento de Física, Facultad de Ciencias, Universidad de Los Andes, \\ Mérida 5101, Venezuela \\ ${ }^{d}$ Centro Nacional de Tecnologías Ópticas (CNTO), Mérida, 5101, Venezeula \\ 'Department of Physics, Sogang University, Seoul, 04017, South Korea
}

\begin{tabular}{|c|c|}
\hline Item & Page(s) \\
\hline Figure S1. Differential thermal analysis diagrams for $\mathrm{Li}_{4} \mathrm{CdSnS}_{7}$. & S2 \\
\hline $\begin{array}{l}\text { Figure } \mathrm{S} 2 . \mathrm{X} \text {-ray powder diffraction pattern for } \mathrm{Li}_{2} \mathrm{CdSiS}_{4} \text { compared to that calculated from the single } \\
\text { crystal X-ray structure. }\end{array}$ & S3 \\
\hline Figure S3. Differential thermal analysis diagrams for $\mathrm{Li}_{2} \mathrm{CdSiS}_{4}$ & S3 \\
\hline $\begin{array}{l}\text { Figure S4. Optical diffuse reflectance spectrum converted to absorption using the Kubelka-Munk } \\
\text { equation for } \mathrm{Li}_{2} \mathrm{CdSiS} \mathrm{S}_{4} \text {. }\end{array}$ & S4 \\
\hline Figure S5. The Brillouin Zone for $\mathrm{Li}_{4} \mathrm{CdSn}{ }_{2} \mathrm{~S}_{7}$ & S5 \\
\hline $\begin{array}{l}\text { Figure S6. (a) SHG and (b) PL spectra from AgGaSe } 2 \text { obtained under various pulse energies, } E_{p}=20.2 \\
\mu \mathrm{J}-236 \mu \mathrm{J} \text { at } \lambda=1064 \mathrm{~nm} \text {. (c) Intensity dependence of SHG (crosses) and PL (triangles) superimposed } \\
\text { by a theoretical fit (red) using Eq. (2). The black line is the case for no fundamental depletion by } 2 \mathrm{PA} \text {. }\end{array}$ & S6 \\
\hline $\begin{array}{l}\text { Figure S7. SHG spectra of } \mathrm{Li}_{4} \mathrm{CdSn}_{2} \mathrm{~S}_{7} \text { obtained under various pulse energies, } E_{p}=20.2 \mu \mathrm{J}-236 \mu \mathrm{J} \text { at } \lambda \\
=1064 \mathrm{~nm} .\end{array}$ & S6 \\
\hline Table S1. Crystal data, data collection and structure refinement details for $\mathrm{Li}_{4} \mathrm{CdSn}_{2} \mathrm{~S}_{7}$ & S7 \\
\hline 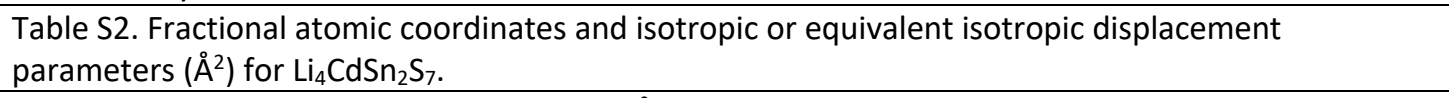 & S8 \\
\hline Table S3. Atomic displacement parameters $\left(\AA^{2}\right)$ for $\mathrm{Li}_{4} \mathrm{CdSn}{ }_{2} \mathrm{~S}_{7}$ & S8 \\
\hline Table S4. Bond distances $(\AA)$ for $\mathrm{Li}_{4} \mathrm{CdSn}_{2} \mathrm{~S}_{7}$ & S9 \\
\hline Table S5. Bond angles $\left({ }^{\circ}\right)$ for $\mathrm{Li}_{4} \mathrm{CdSn}_{2} \mathrm{~S}_{7}$ & S10 \\
\hline $\begin{array}{l}\text { Table S6. Extended connectivity table for } \mathrm{Li}_{4} \mathrm{CdSn}_{2} \mathrm{~S}_{7} \text { used to predict structural distortions according } \\
\text { to Pauling's second rule. }\end{array}$ & S11 \\
\hline $\begin{array}{l}\text { Table S7. Calculation of dipole moments, expressed in units of Debye (D), for each tetrahedron and } \\
\text { net dipole moment for } \mathrm{Li}_{4} \mathrm{CdSn} \mathrm{S}_{7} \text {. }\end{array}$ & S12 \\
\hline $\begin{array}{l}\text { Table S8. Calculation of dipole moments, expressed in units of Debye (D), for each tetrahedron and } \\
\text { net dipole moment for } \mathrm{Li}_{2} \mathrm{CdSiS}_{4} \text {. }\end{array}$ & S13 \\
\hline $\begin{array}{l}\text { Table S9. Calculation of dipole moments, expressed in units of Debye (D), for each tetrahedron and } \\
\text { net dipole moment for } \mathrm{LiGaS}_{2} \text {. }\end{array}$ & S13 \\
\hline $\begin{array}{l}\text { Table S10. Calculation of dipole moments, expressed in units of Debye (D), for each tetrahedron and } \\
\text { net dipole moment for LilnS } 2 .\end{array}$ & S14 \\
\hline $\begin{array}{l}\text { Table S11. Calculation of dipole moments, expressed in units of Debye (D), for each tetrahedron and } \\
\text { net dipole moment for LilnSe. }\end{array}$ & S14 \\
\hline Table S12. The calculated Mulliken bond populations for $\mathrm{Li}_{4} \mathrm{CdSn}_{2} \mathrm{~S}_{7}$ & S15 \\
\hline $\begin{array}{l}\text { Table S13. Chalcogenides for which both second harmonic generation and laser-induced damage } \\
\text { threshold (LIDT) have been reported. }\end{array}$ & S16-S18 \\
\hline Supporting Information references & S19-S21 \\
\hline
\end{tabular}



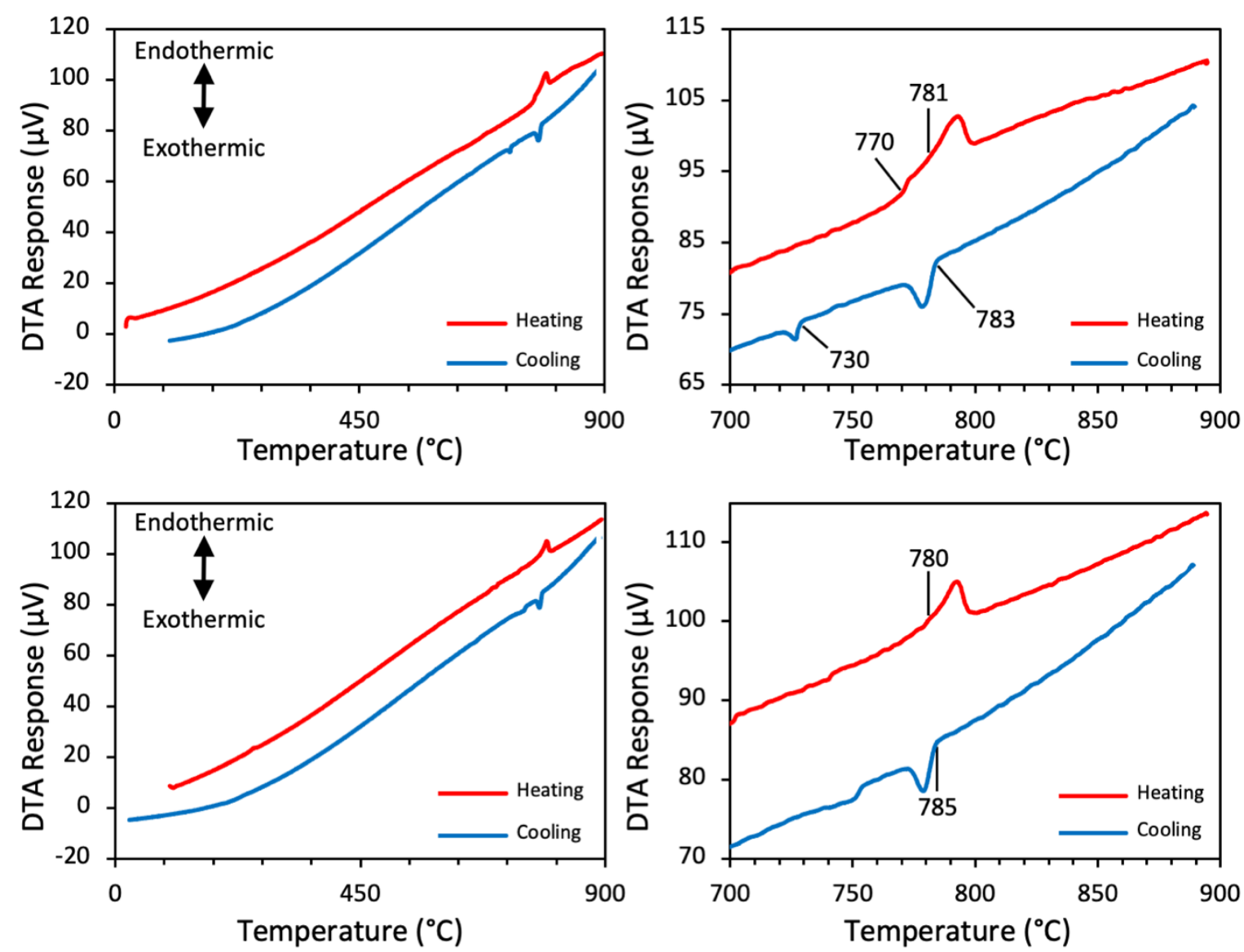

Figure S1. Differential thermal analysis diagram for $\mathrm{Li}_{4} \mathrm{CdS} \mathrm{n}_{2} \mathrm{~S}_{7}$. The two plots above represent the heating and cooling curves for cycle 1 (top) and cycle 2 (bottom) of the same experiment. 


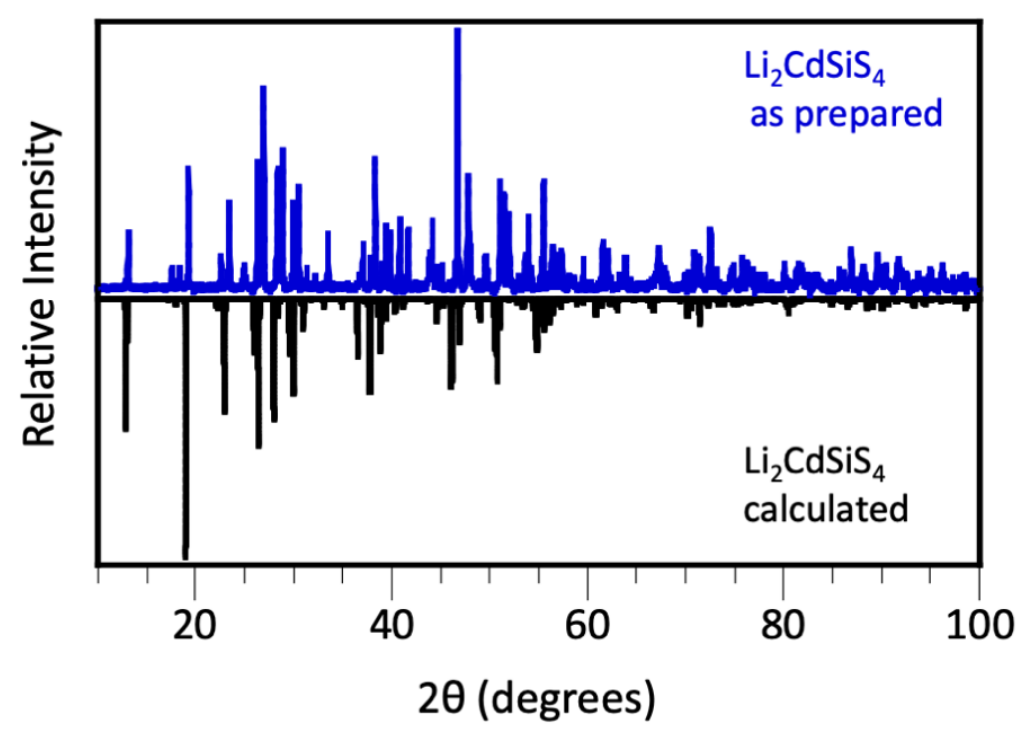

Figure S2. X-ray powder diffraction pattern for $\mathrm{Li}_{2} \mathrm{CdSiS}_{4}$ compared to that calculated from the single crystal X-ray structure.
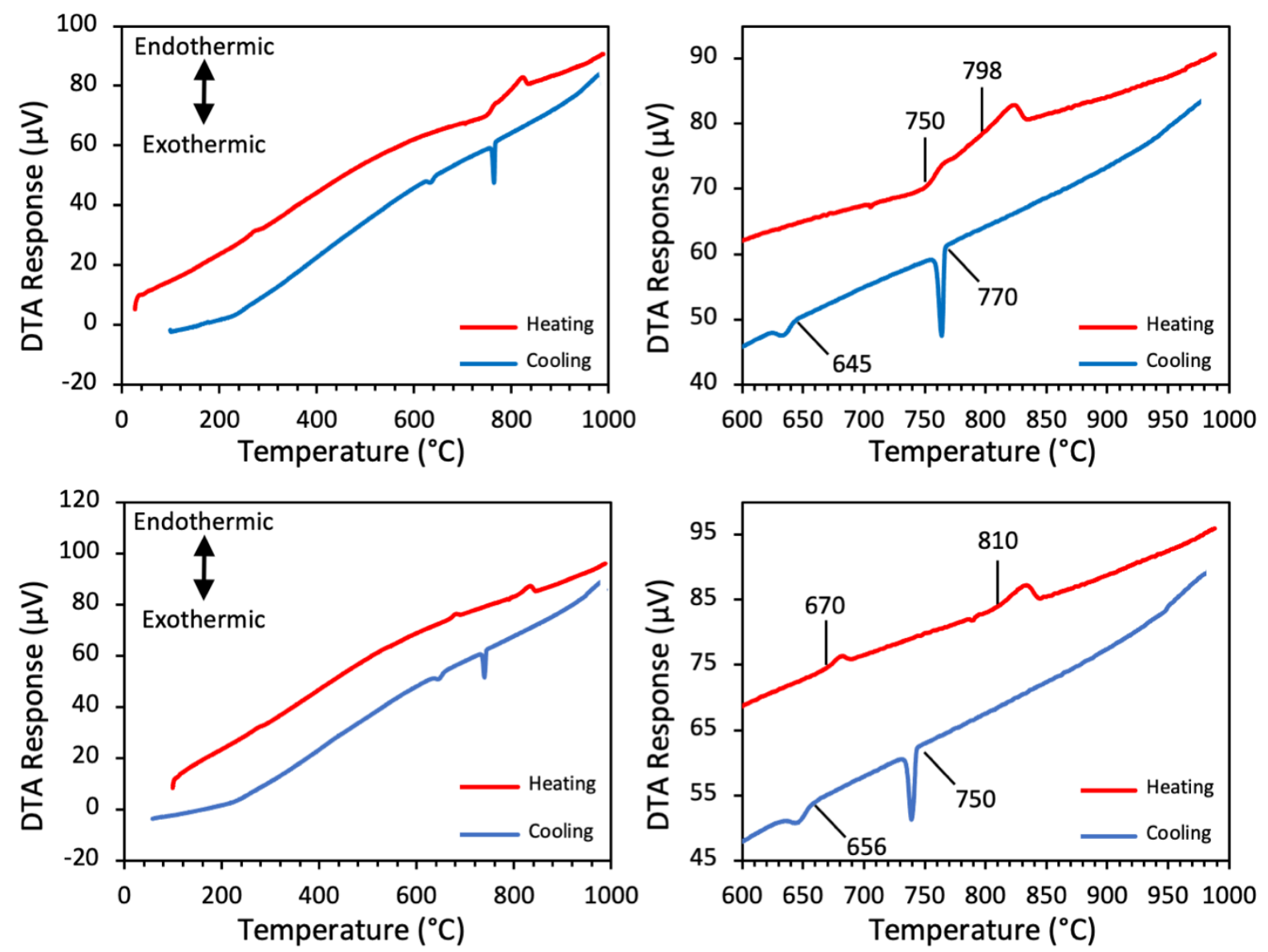

Figure S3. Differential thermal analysis diagram for $\mathrm{Li}_{2} \mathrm{CdSiS}_{4}$. The two plots above represent the heating and cooling curves for cycle 1 (top) and cycle 2 (bottom) of the same experiment. 

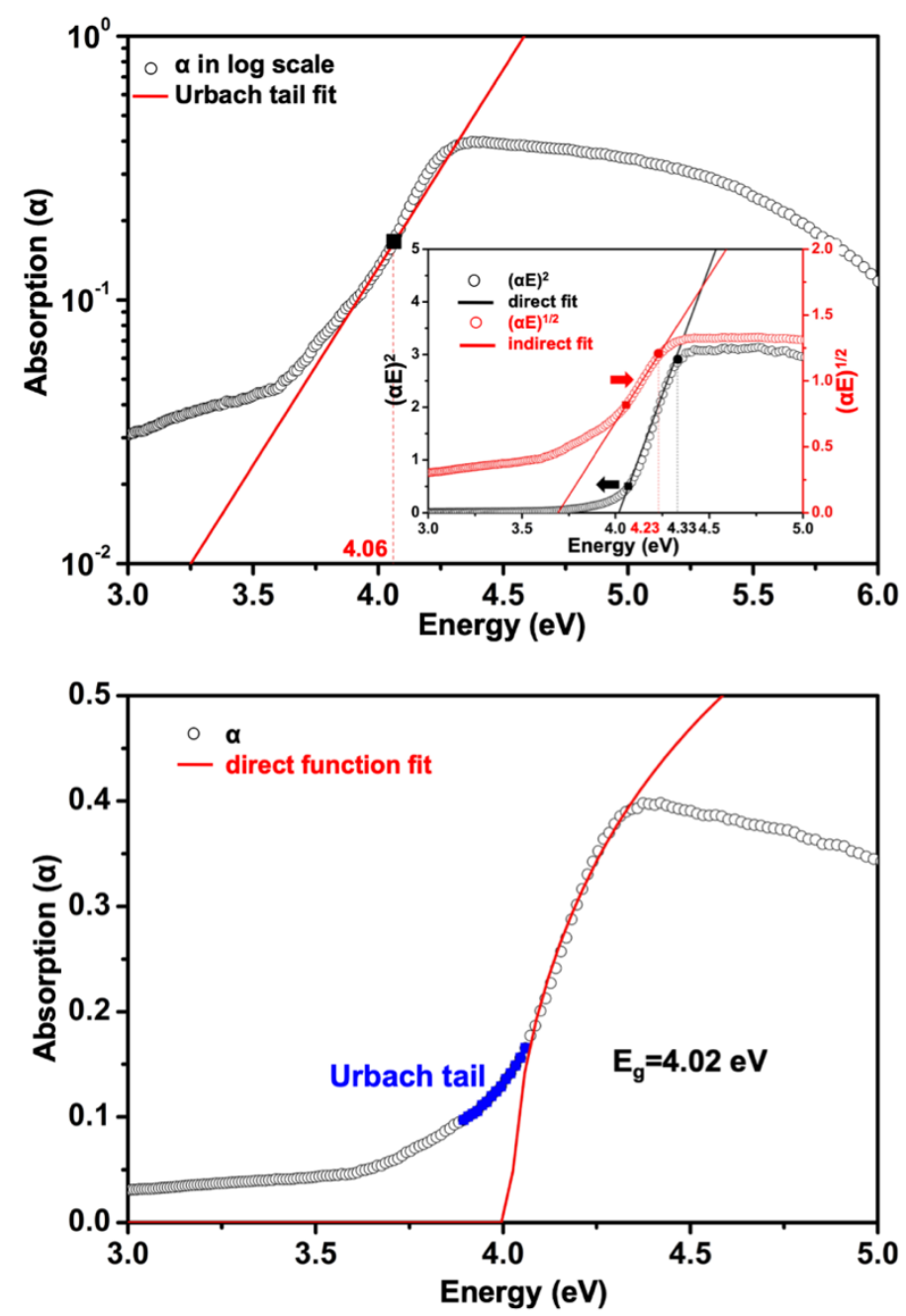

Figure S4. Optical diffuse reflectance spectrum converted to absorption using the Kubelka-Munk equation for $\mathrm{Li}_{2} \mathrm{CdSiS}_{4}$. The log of the absorption is shown on top. The linear region in the log plot was identified as the Urbach tail and excluded when determining the nature of the gap (i.e. direct vs. indirect). The inset on the top shows Tauc's function for direct and indirect gap semiconductors. A wider region of energy could be fit using the direct function. The bottom shows the fit for the direct function yielding an $\mathrm{E}_{\mathrm{g}}$ value of $4.02 \mathrm{eV}$. This value is significantly wider than that reported by Li et. al. ( $3.76 \mathrm{eV}$ ), which they simply determined by extrapolation of the absorption edge to the baseline. The difference in the bandgap could be due to the difference in the bandgap determination procedure or, more likely, the difference in the synthetic procedures. 


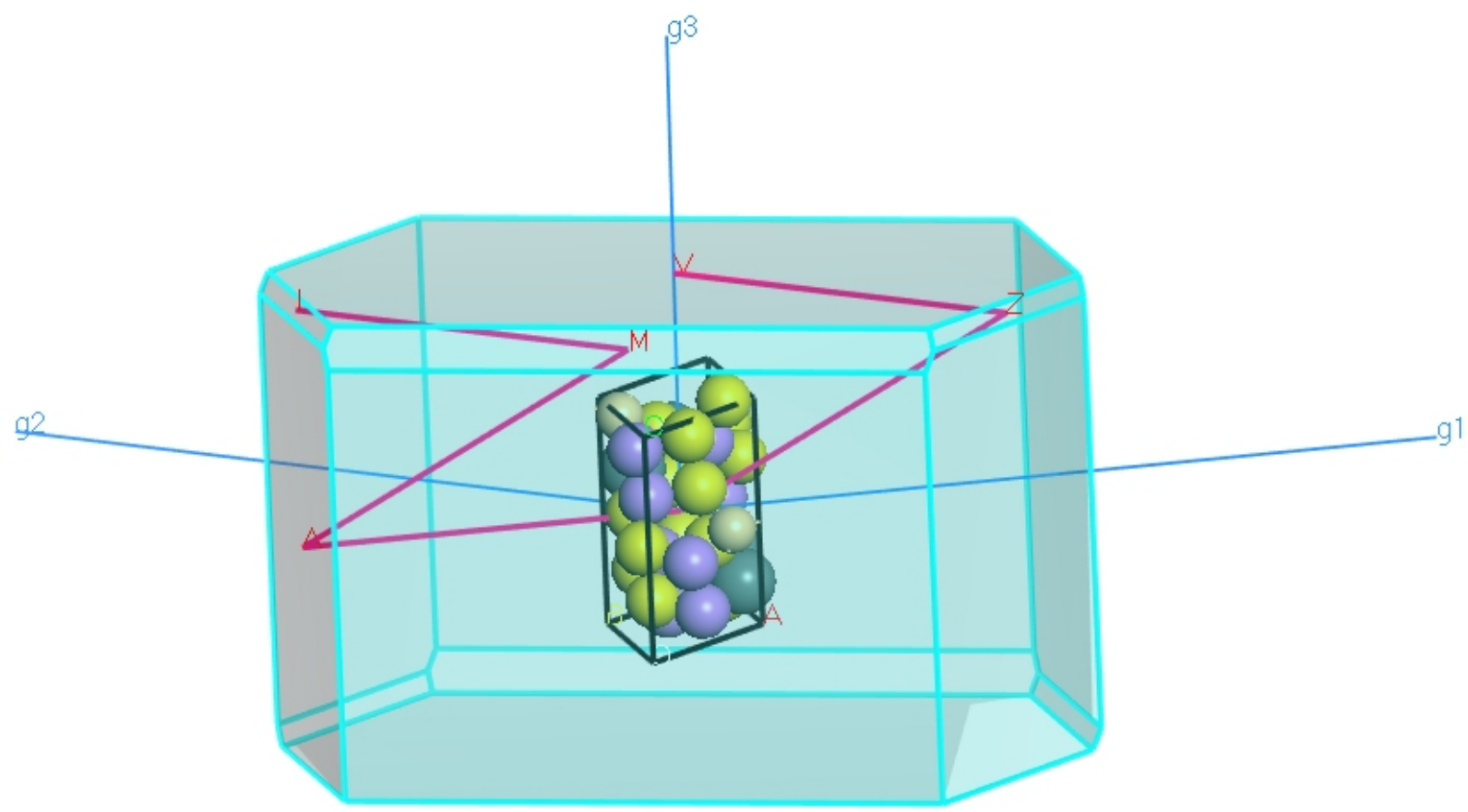

Figure S5. The Brillouin zone for $\mathrm{Li}_{4} \mathrm{CdSn}_{2} \mathrm{~S}_{7}$. 


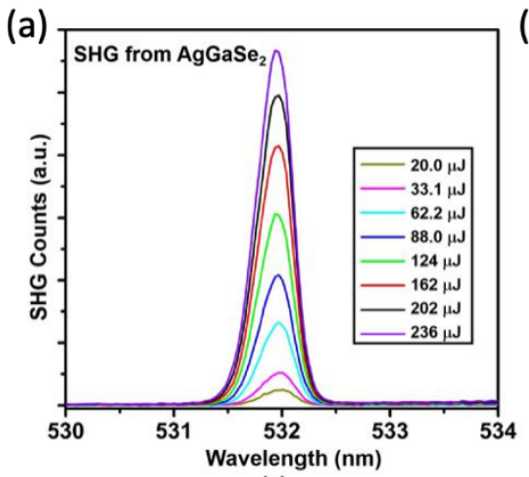

(a)

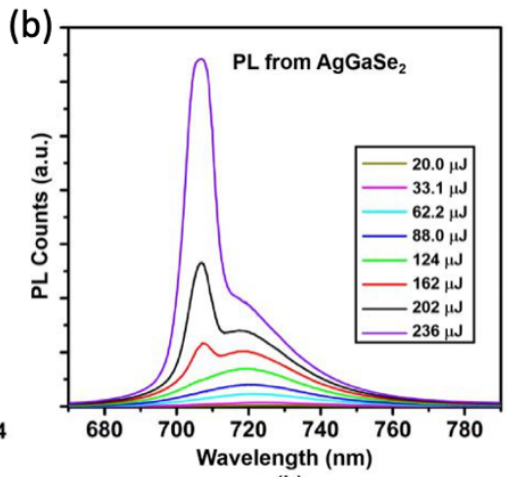

(b)

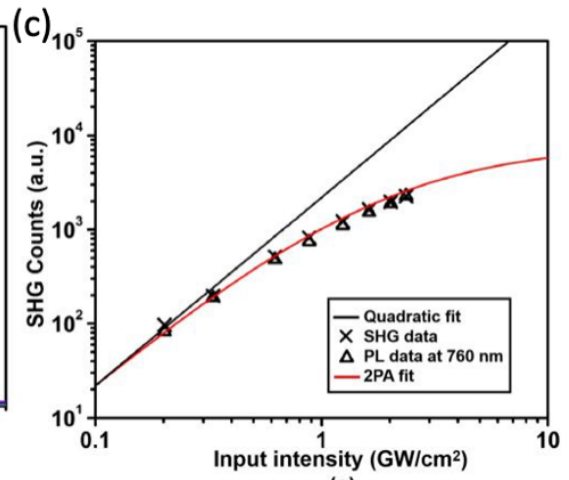

Figure S6. (a) SHG and (b) PL spectra from AgGaSe 2 obtained under various pulse energies, $E_{p}=20.2 \mu \mathrm{J}-236 \mu \mathrm{J}$ at $\lambda$ $=1064 \mathrm{~nm}$. (c) Intensity dependence of SHG (crosses) and PL (triangles) superimposed by a theoretical fit (red) using Eq. (2). The PL counts are scaled to match with the SHG counts to check the trend of intensity dependence. The black line is the case for no fundamental depletion by $2 \mathrm{PA}$.

Figure S6 discussion: For the $\mathrm{AgGaSe}_{2}$ reference material, we observed highly efficient PL emission driven by twophoton absorption (2PA), where the emission edge is consistent with its bandgap energy of $\sim 1.8 \mathrm{eV}(\sim 690 \mathrm{~nm})$. Interestingly, the compound exhibits amplified spontaneous emission (ASE) when the intensity is larger than 0.9 $\mathrm{GW} / \mathrm{cm}^{2}$ as evidenced by super-linear increase in the peak near $\sim 705 \mathrm{~nm}$ with a threshold behavior. This implies that $\mathrm{AgGaSe}_{2}$ is a good gain medium when employed under laser cavity confinement. ${ }^{1}$ The corresponding SHG counts (cross) and the PL counts (triangles) almost overlap each other with a proper scaling as shown in Fig. S6(c); here the PL counts were measured at $760 \mathrm{~nm}$ where the contribution by ASE is negligible.

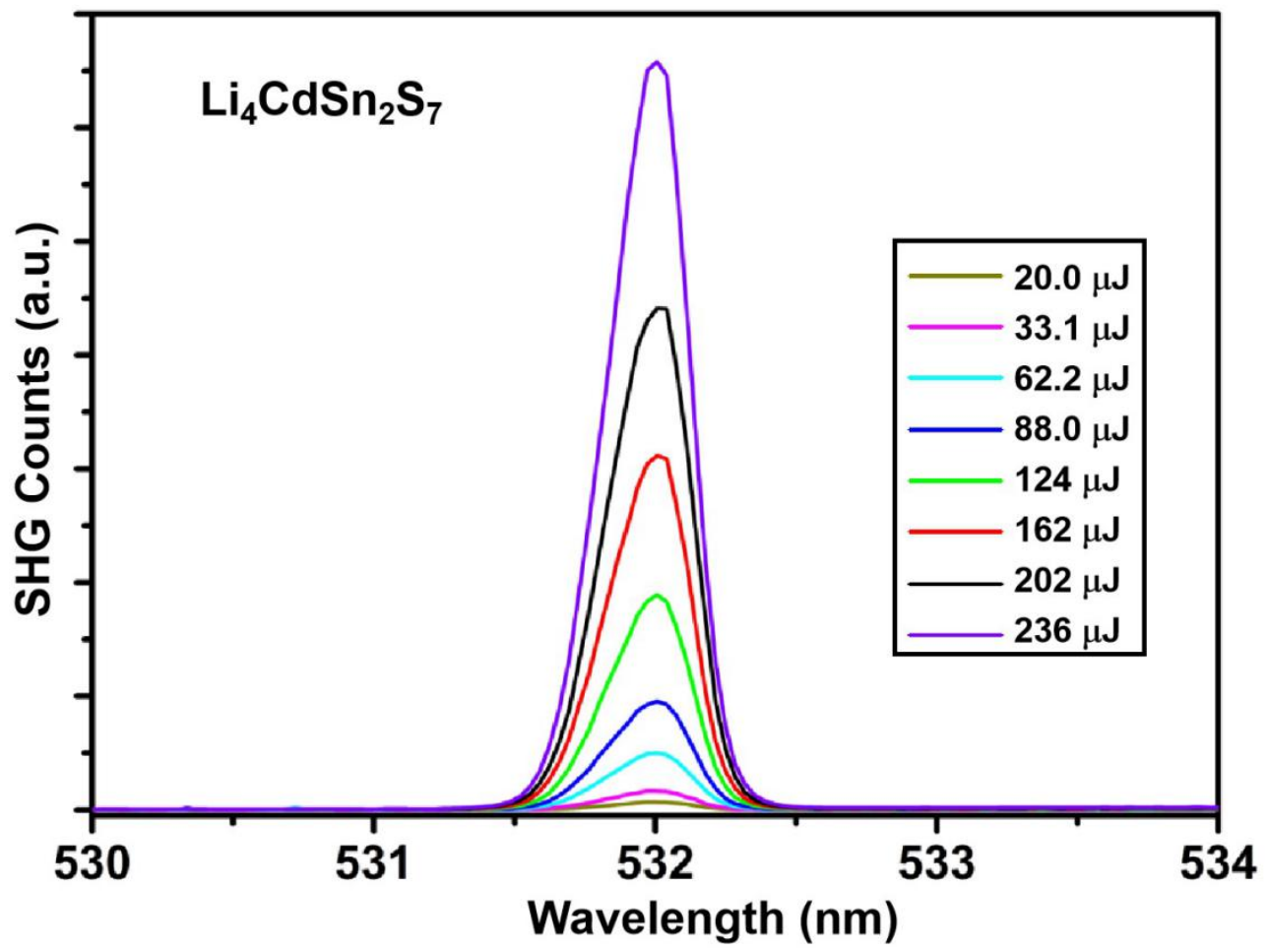

Figure S7. SHG spectra of $\mathrm{Li}_{4} \mathrm{CdSn}{ }_{2} \mathrm{~S}_{7}$ obtained under various pulse energies, $E_{p}=20.2 \mu \mathrm{J}-236 \mu \mathrm{J}$ at $\lambda=1064 \mathrm{~nm}$. 
Table S1. Crystal data, data collection and structure refinement details for $\mathrm{Li}_{4} \mathrm{CdSn}_{2} \mathrm{~S}_{7}$.

\begin{tabular}{|c|c|}
\hline Formula weight & 601.96 \\
\hline Crystal system, space group & Monoclinic, Cc (No.9) \\
\hline Temperature $(\mathrm{K})$ & 296 \\
\hline$a(\AA)$ & $17.4430(2)$ \\
\hline$b(\AA)$ & $6.93532(7)$ \\
\hline$c(\AA)$ & $10.3271(1)$ \\
\hline$\beta\left(^{\circ}\right)$ & 93.9042(5) \\
\hline$V\left(\AA^{3}\right)$ & $1246.39(2)$ \\
\hline$Z$ & 4 \\
\hline$F(000)$ & 1088 \\
\hline Density $\mathrm{g} \mathrm{cm}^{-3}$ & 3.208 \\
\hline Radiation type & Mo $K \alpha$ \\
\hline$\mu\left(\mathrm{mm}^{-1}\right)$ & 6.78 \\
\hline Crystal size $(\mathrm{mm})$ & $0.15 \times 0.10 \times 0.09$ \\
\hline Crystal habit and color & Irregular polyhedron, pale yellow \\
\hline Diffractometer & Bruker SMART Apex II \\
\hline Radiation source & Fine-focus sealed tube \\
\hline Absorption correction & Multi-scan, SADABS (Sheldrick, 2002) \\
\hline No. of measured reflections & 5186 \\
\hline No. of independent reflections & 2839 \\
\hline No. of observed reflections $[/>2 \sigma(/)]$ & 2801 \\
\hline$\theta_{\min }, \theta_{\max }\left(^{\circ}\right)$ & $2.3,27.5$ \\
\hline Completeness to $\theta=27.476^{\circ}$ & $100 \%$ \\
\hline Limiting indices & $\begin{array}{l}-22 \leq h \leq 22 \\
-8 \leq k \leq 8 \\
-13 \leq 1 \leq 13\end{array}$ \\
\hline$R_{\text {int }}$ & 0.011 \\
\hline$R\left[F^{2}>2 \sigma\left(F^{2}\right)\right], \mathrm{wR}\left(F^{2}\right),{ }^{*} S$ & $0.014,0.039,1.13$ \\
\hline$(\Delta / \sigma)_{\max }$ & 0.001 \\
\hline Extinction coefficient & $0.00182(7)$ \\
\hline No. of data/restraints/parameters & $2839 / 129 / 2$ \\
\hline$\Delta \rho_{\max } \Delta \rho_{\min }\left(\mathrm{e} \AA^{-3}\right)$ & $0.48,-0.93$ \\
\hline Absolute structure parameter (Flack parameter) & $0.06(3)$ \\
\hline
\end{tabular}


Table S2. Fractional atomic coordinates and isotropic or equivalent isotropic displacement parameters $\left(\AA^{2}\right)$ for $\mathrm{Li}_{4} \mathrm{CdSn}_{2} \mathrm{~S}_{7}$.

\begin{tabular}{lllll}
\hline & $x$ & $y$ & $z$ & $U_{\text {iso }} * / U_{\text {eq }}$ \\
\hline Li1 & $0.8347(16)$ & $0.679(2)$ & $0.498(3)$ & $0.027(6)$ \\
Li2 & $0.625(2)$ & $0.828(2)$ & $0.405(4)$ & $0.039(10)$ \\
Li3 & $0.4787(16)$ & $0.8268(11)$ & $0.699(4)$ & $0.014(5)$ \\
Li4 & $0.2679(11)$ & $0.6617(18)$ & $0.639(3)$ & $0.015(5)$ \\
Cd & $0.54874(11)$ & $0.34736(6)$ & $0.5638(2)$ & $0.02106(14)$ \\
Sn1 & $0.90772(2)$ & $0.83959(7)$ & $0.84366(3)$ & $0.01293(19)$ \\
Sn2 & $0.69288(2)$ & $0.67320(7)$ & $0.77412(4)$ & $0.01259(17)$ \\
S1 & $1.00959(8)$ & $0.80885(18)$ & $1.00890(16)$ & $0.0160(3)$ \\
S2 & $0.94103(10)$ & $0.66013(18)$ & $0.65885(17)$ & $0.0160(3)$ \\
S3 & $0.87228(10)$ & $0.83835(19)$ & $0.30415(18)$ & $0.0178(3)$ \\
S4 & $0.80192(9)$ & $0.66569(18)$ & $0.93812(16)$ & $0.0141(3)$ \\
S5 & $0.72681(9)$ & $0.8370(2)$ & $0.58809(18)$ & $0.0173(3)$ \\
S6 & $0.66230(8)$ & $0.34428(17)$ & $0.73087(16)$ & $0.0156(3)$ \\
S7 & $0.58956(8)$ & $0.83567(19)$ & $0.86668(17)$ & $0.0158(3)$ \\
\hline
\end{tabular}

Table S3. Atomic displacement parameters $\left(\AA^{2}\right)$ for $\mathrm{Li}_{4} \mathrm{CdSn}_{2} \mathrm{~S}_{7}$.

\begin{tabular}{lllllll}
\hline & $U^{11}$ & $U^{22}$ & $U^{33}$ & $U^{12}$ & $U^{13}$ & $U^{23}$ \\
\hline Li1 & $0.028(10)$ & $0.042(11)$ & $0.010(8)$ & $-0.008(6)$ & $0.004(6)$ & $0.007(6)$ \\
Li2 & $0.045(15)$ & $0.032(11)$ & $0.039(18)$ & $0.003(6)$ & $0.003(11)$ & $-0.005(6)$ \\
Li3 & $0.011(7)$ & $0.013(7)$ & $0.018(9)$ & $-0.005(6)$ & $0.000(6)$ & $0.004(6)$ \\
Li4 & $0.006(7)$ & $0.019(8)$ & $0.022(10)$ & $-0.005(5)$ & $0.007(6)$ & $-0.004(5)$ \\
Cd & $0.0208(2)$ & $0.02254(19)$ & $0.0200(3)$ & $0.0003(3)$ & $0.00275(18)$ & $-0.0002(3)$ \\
Sn1 & $0.0121(3)$ & $0.0131(3)$ & $0.0136(4)$ & $-0.0006(2)$ & $0.0007(2)$ & $0.0003(2)$ \\
Sn2 & $0.0114(3)$ & $0.0138(2)$ & $0.0126(3)$ & $-0.00088(19)$ & $0.0007(2)$ & $0.0000(2)$ \\
S1 & $0.0146(6)$ & $0.0177(6)$ & $0.0152(7)$ & $0.0003(5)$ & $-0.0019(5)$ & $0.0003(6)$ \\
S2 & $0.0190(7)$ & $0.0152(6)$ & $0.0141(7)$ & $-0.0005(5)$ & $0.0033(6)$ & $-0.0015(5)$ \\
S3 & $0.0198(8)$ & $0.0151(6)$ & $0.0184(7)$ & $-0.0015(5)$ & $0.0007(6)$ & $-0.0007(5)$ \\
S4 & $0.0120(5)$ & $0.0170(6)$ & $0.0133(7)$ & $-0.0017(5)$ & $-0.0001(5)$ & $0.0007(5)$ \\
S5 & $0.0181(7)$ & $0.0195(7)$ & $0.0146(7)$ & $-0.0024(5)$ & $0.0024(6)$ & $0.0034(5)$ \\
S6 & $0.0158(7)$ & $0.0138(6)$ & $0.0172(7)$ & $-0.0010(5)$ & $0.0000(6)$ & $-0.0011(5)$ \\
S7 & $0.0147(6)$ & $0.0161(6)$ & $0.0167(7)$ & $0.0019(5)$ & $0.0031(5)$ & $0.0001(5)$ \\
\hline
\end{tabular}


Table S4. Bond distances ( $\AA$ ) for $\mathrm{Li}_{4} \mathrm{CdSn}_{2} \mathrm{~S}_{7}$.

\begin{tabular}{|c|c|c|c|}
\hline $\mathrm{Li} 1-\mathrm{S} 2$ & $2.41(3)$ & $\mathrm{Li} 4-\mathrm{S}^{\mathrm{iv}}$ & 2.478 (19) \\
\hline $\mathrm{Li} 1-\mathrm{S} 3$ & $2.41(3)$ & $\mathrm{Li} 4-\mathrm{S} 4^{\mathrm{iii}}$ & $2.50(3)$ \\
\hline Li1-S5 & $2.42(3)$ & $\mathrm{Cd}-\mathrm{S} 1^{\mathrm{iii}}$ & $2.5336(16)$ \\
\hline $\mathrm{Li} 1-\mathrm{S} 4^{\mathrm{i}}$ & $2.527(18)$ & $\mathrm{Cd}-\mathrm{S} 2^{\mathrm{vi}}$ & $2.536(2)$ \\
\hline $\mathrm{Li} 2-\mathrm{S}^{\mathrm{i}}$ & $2.29(4)$ & $\mathrm{Cd}-\mathrm{S} 6$ & $2.537(2)$ \\
\hline $\mathrm{Li} 2-\mathrm{S} 7^{\mathrm{ii}}$ & $2.43(2)$ & $\mathrm{Cd}-\mathrm{S}^{\mathrm{i}}$ & $2.541(2)$ \\
\hline $\mathrm{Li} 2-\mathrm{S} 5$ & $2.51(4)$ & $\mathrm{Sn} 1-\mathrm{S} 3^{\mathrm{vii}}$ & $2.3459(13)$ \\
\hline $\mathrm{Li} 2-\mathrm{S} 1^{\mathrm{iii}}$ & $2.53(4)$ & $\mathrm{Sn} 1-\mathrm{S} 2$ & $2.3834(18)$ \\
\hline $\mathrm{Li3}-\mathrm{S} 1^{\mathrm{iii}}$ & $2.27(3)$ & $\mathrm{Sn} 1-\mathrm{S} 1$ & $2.3878(16)$ \\
\hline $\mathrm{Li} 3-\mathrm{S} 2^{\mathrm{iv}}$ & $2.431(12)$ & $\mathrm{Sn} 1-\mathrm{S} 4$ & $2.4611(16)$ \\
\hline $\mathrm{Li} 3-\mathrm{S}^{\mathrm{v}}$ & $2.49(3)$ & $\mathrm{Sn} 2-\mathrm{S} 5$ & $2.3423(19)$ \\
\hline $\mathrm{Li3}-\mathrm{S} 7$ & $2.51(3)$ & $\mathrm{Sn} 2-\mathrm{S} 6$ & $2.3781(12)$ \\
\hline $\mathrm{Li} 4-\mathrm{S}^{\mathrm{v}}$ & $2.41(3)$ & $\mathrm{Sn} 2-\mathrm{S} 7$ & $2.3802(16)$ \\
\hline $\mathrm{Li} 4-\mathrm{S}^{\mathrm{vi}}$ & $2.410(13)$ & $\mathrm{Sn} 2-\mathrm{S} 4$ & $2.4598(18)$ \\
\hline
\end{tabular}

Symmetry codes: (i) $x,-y+1, z-1 / 2$; (ii) $x,-y+2, z-1 / 2$; (iii) $x-1 / 2,-y+3 / 2, z-1 / 2$; (iv) $x-1 / 2, y+1 / 2, z$; (v) $x-1 / 2,-y+3 / 2, z+1 / 2$; (vi) $x-1 / 2, y-1 / 2, z$; (vii) $x,-y+2, z+1 / 2$; (viii) $x+1 / 2,-y+3 / 2, z+1 / 2$; (ix) $x+1 / 2, y-1 / 2, z$; (x) $x+1 / 2, y+1 / 2, z$; (xi) $x+1 / 2,-y+3 / 2, z-1 / 2$; (xii) $x,-y+1, z+1 / 2$. 
Table S5. Bond angles ( ${ }^{\circ}$ ) for $\mathrm{Li}_{4} \mathrm{CdSn}_{2} \mathrm{~S}_{7}$.

\begin{tabular}{|c|c|c|c|}
\hline $\mathrm{S} 2-\mathrm{Li} 1-\mathrm{S} 3$ & $111.0(10)$ & $\mathrm{Li} 3^{\text {viii }}-\mathrm{S} 1-\mathrm{Sn} 1$ & $116.4(7)$ \\
\hline $\mathrm{S} 2-\mathrm{Li} 1-\mathrm{S} 5$ & $109.9(11)$ & $\mathrm{Li} 3^{\text {viii }}-\mathrm{S} 1-\mathrm{Li} 2^{\text {viii }}$ & $116.9(9)$ \\
\hline $\mathrm{S} 3-\mathrm{Li} 1-\mathrm{S} 5$ & $112.1(9)$ & $\mathrm{Sn} 1-\mathrm{S} 1-\mathrm{Li} 2^{\mathrm{viii}}$ & $107.7(10)$ \\
\hline $\mathrm{S} 2-\mathrm{Li} 1-\mathrm{S} 4^{\mathrm{i}}$ & $105.4(8)$ & $\mathrm{Li} 3^{\text {viii }}-\mathrm{S} 1-\mathrm{Cd}^{\text {viii }}$ & $105.8(4)$ \\
\hline $\mathrm{S} 3-\mathrm{Li} 1-\mathrm{S} 4^{\mathrm{i}}$ & $107.5(11)$ & $\mathrm{Sn} 1-\mathrm{S} 1-\mathrm{Cd}^{\mathrm{viii}}$ & $104.59(6)$ \\
\hline $\mathrm{S} 5-\mathrm{Li} 1-\mathrm{S} 4^{\mathrm{i}}$ & $110.7(10)$ & $\mathrm{Li} 2^{\mathrm{viii}}-\mathrm{S} 1-\mathrm{Cd}^{\mathrm{viii}}$ & $103.8(5)$ \\
\hline $\mathrm{S} 6{ }^{\mathrm{i}}-\mathrm{Li} 2-\mathrm{S} 7^{\mathrm{ii}}$ & $117.1(18)$ & $\mathrm{Sn} 1-\mathrm{S} 2-\mathrm{Li} 1$ & $107.8(6)$ \\
\hline $\mathrm{S} 66^{\mathrm{i}-\mathrm{Li} 2-\mathrm{S} 5}$ & $112.3(14)$ & $\mathrm{Sn} 1-\mathrm{S} 2-\mathrm{Li} 3^{\mathrm{ix}}$ & $116.0(9)$ \\
\hline $\mathrm{S} 7 \mathrm{ii}-\mathrm{Li} 2-\mathrm{S} 5$ & $105.0(10)$ & $\mathrm{Li} 1-\mathrm{S} 2-\mathrm{Li} 3^{\mathrm{ix}}$ & $110.9(7)$ \\
\hline $\mathrm{S} 6^{\mathrm{i}}-\mathrm{Li} 2-\mathrm{S} 1^{\mathrm{iii}}$ & $114.4(10)$ & $\mathrm{Sn} 1-\mathrm{S} 2-\mathrm{Cd}^{\mathrm{x}}$ & $105.67(6)$ \\
\hline $\mathrm{S} 7^{\mathrm{ii}}-\mathrm{Li} 2-\mathrm{S} 1^{\mathrm{iii}}$ & $103.2(13)$ & $\mathrm{Li} 1-\mathrm{S} 2-\mathrm{Cd}^{\mathrm{x}}$ & $105.1(6)$ \\
\hline $\mathrm{S} 5-\mathrm{Li} 2-\mathrm{S} 1^{\mathrm{iii}}$ & $103.4(16)$ & $\mathrm{Li}^{3}{ }^{\mathrm{ix}}-\mathrm{S} 2-\mathrm{Cd}^{\mathrm{x}}$ & $110.7(7)$ \\
\hline 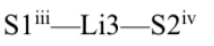 & $108.9(13)$ & $\mathrm{Sn} 1^{\mathrm{ii}}-\mathrm{S} 3-\mathrm{Li} 4^{\mathrm{xi}}$ & $107.5(3)$ \\
\hline 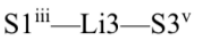 & $114.4(7)$ & $\mathrm{Sn} 1^{\mathrm{ii}}-\mathrm{S} 3-\mathrm{Li} 1$ & $112.0(5)$ \\
\hline $\mathrm{S} 2^{\mathrm{iv}}-\mathrm{Li} 3-\mathrm{S}^{\mathrm{v}}$ & $108.0(11)$ & $\mathrm{Li}^{\mathrm{xi}}-\mathrm{S} 3-\mathrm{Li} 1$ & $110.6(9)$ \\
\hline $\mathrm{S} 1^{\mathrm{iii}-\mathrm{Li} 3-\mathrm{S} 7}$ & $113.0(11)$ & $\mathrm{Sn} 1^{\mathrm{ii}}-\mathrm{S} 3-\mathrm{Li} 3^{\mathrm{xi}}$ & $108.5(4)$ \\
\hline $\mathrm{S} 2^{\mathrm{iv}}-\mathrm{Li} 3-\mathrm{S} 7$ & $106.3(7)$ & $\mathrm{Li}^{\mathrm{xi}}-\mathrm{S} 3-\mathrm{Li}^{3 \mathrm{xi}}$ & $103.9(10)$ \\
\hline $\mathrm{S} 3^{\mathrm{v}}-\mathrm{Li} 3-\mathrm{S} 7$ & $105.8(13)$ & $\mathrm{Li} 1-\mathrm{S} 3-\mathrm{Li}^{\mathrm{xi}}$ & $113.8(8)$ \\
\hline $\mathrm{S} 3^{\mathrm{v}}-\mathrm{Li} 4-\mathrm{S} 5^{\mathrm{vi}}$ & $110.7(8)$ & $\mathrm{Sn} 2-\mathrm{S} 4-\mathrm{Sn} 1$ & $106.39(6)$ \\
\hline $\mathrm{S} 3^{\mathrm{v}}-\mathrm{Li} 4-\mathrm{S} 6^{\mathrm{iv}}$ & $105.9(10)$ & $\mathrm{Sn} 2-\mathrm{S} 4-\mathrm{Li}^{\text {viii }}$ & $110.2(5)$ \\
\hline $\mathrm{S} 5^{\mathrm{vi}}-\mathrm{Li} 4-\mathrm{S} 6^{\mathrm{iv}}$ & $110.1(7)$ & $\mathrm{Sn} 1-\mathrm{S} 4-\mathrm{Li}^{\text {viii }}$ & $108.7(4)$ \\
\hline $\mathrm{S} 3^{\mathrm{v}}-\mathrm{Li} 4-\mathrm{S} 4^{\mathrm{iii}}$ & $112.1(7)$ & $\mathrm{Sn} 2-\mathrm{S} 4-\mathrm{Li} 1^{\mathrm{xii}}$ & $109.8(6)$ \\
\hline $\mathrm{S} 5^{\mathrm{vi}}-\mathrm{Li} 4-\mathrm{S} 4^{\mathrm{iii}}$ & $110.6(10)$ & $\mathrm{Sn} 1-\mathrm{S} 4-\mathrm{Li1}^{\mathrm{xii}}$ & $113.4(6)$ \\
\hline $\mathrm{S} 6^{\mathrm{iv}}-\mathrm{Li} 4-\mathrm{S} 4^{\mathrm{iii}}$ & $107.3(7)$ & $\mathrm{Li} 4^{\mathrm{viii}}-\mathrm{S} 4-\mathrm{Li} 1^{\mathrm{xii}}$ & $108.3(10)$ \\
\hline $\mathrm{S} 1^{\mathrm{iii}}-\mathrm{Cd}-\mathrm{S} 2^{\mathrm{vi}}$ & $111.90(8)$ & $\mathrm{Sn} 2-\mathrm{S} 5-\mathrm{Li}^{\mathrm{x}}$ & $111.3(7)$ \\
\hline $\mathrm{S} 1^{\mathrm{iii}}-\mathrm{Cd}-\mathrm{S} 6$ & $110.25(7)$ & $\mathrm{Sn} 2-\mathrm{S} 5-\mathrm{Li} 1$ & $110.1(6)$ \\
\hline $\mathrm{S} 2{ }^{\mathrm{vi}}-\mathrm{Cd}-\mathrm{S} 6$ & $107.28(9)$ & $\mathrm{Li} 4^{\mathrm{x}}-\mathrm{S} 5-\mathrm{Li} 1$ & $106.2(8)$ \\
\hline $\mathrm{S} 1^{\mathrm{iii}}-\mathrm{Cd}-\mathrm{S} 7^{\mathrm{i}}$ & $112.14(9)$ & $\mathrm{Sn} 2-\mathrm{S} 5-\mathrm{Li} 2$ & $113.7(9)$ \\
\hline $\mathrm{S} 2^{\mathrm{vi}}-\mathrm{Cd}-\mathrm{S} 7^{\mathrm{i}}$ & $108.04(5)$ & $\mathrm{Li} 4^{\mathrm{x}}-\mathrm{S} 5-\mathrm{Li} 2$ & $111.8(8)$ \\
\hline $\mathrm{S} 6-\mathrm{Cd}-\mathrm{S} 7^{\mathrm{i}}$ & $106.99(7)$ & $\mathrm{Li} 1-\mathrm{S} 5-\mathrm{Li} 2$ & $103.2(12)$ \\
\hline $\mathrm{S} 3^{\mathrm{vii}}-\mathrm{Sn} 1-\mathrm{S} 2$ & $115.70(7)$ & $\mathrm{Li} 2^{\mathrm{xii}}-\mathrm{S} 6-\mathrm{Sn} 2$ & $115.2(8)$ \\
\hline $\mathrm{S} 3^{\mathrm{vii}}-\mathrm{Sn} 1-\mathrm{S} 1$ & $112.57(5)$ & $\mathrm{Li}^{\mathrm{xii}}-\mathrm{S} 6-\mathrm{Li} 4^{\mathrm{ix}}$ & $107.0(9)$ \\
\hline $\mathrm{S} 2-\mathrm{Sn} 1-\mathrm{S} 1$ & $108.39(6)$ & $\mathrm{Sn} 2-\mathrm{S} 6-\mathrm{Li} 4^{\mathrm{ix}}$ & $113.5(3)$ \\
\hline $\mathrm{S} 3^{\mathrm{vii}}-\mathrm{S} n 1-\mathrm{S} 4$ & $109.86(6)$ & $\mathrm{Li} 2^{\mathrm{xii}}-\mathrm{S} 6-\mathrm{Cd}$ & $106.7(10)$ \\
\hline $\mathrm{S} 2-\mathrm{Sn} 1-\mathrm{S} 4$ & $106.96(5)$ & $\mathrm{Sn} 2-\mathrm{S} 6-\mathrm{Cd}$ & $105.93(6)$ \\
\hline $\mathrm{S} 1-\mathrm{Sn} 1-\mathrm{S} 4$ & $102.39(6)$ & $\mathrm{Li} 4^{\mathrm{ix}}-\mathrm{S} 6-\mathrm{Cd}$ & $108.0(6)$ \\
\hline $\mathrm{S} 5-\mathrm{Sn} 2-\mathrm{S} 6$ & $112.15(7)$ & $\mathrm{Sn} 2-\mathrm{S} 7-\mathrm{Li} 2^{\mathrm{vii}}$ & $109.1(10)$ \\
\hline $\mathrm{S} 5-\mathrm{Sn} 2-\mathrm{S} 7$ & $109.90(6)$ & $\mathrm{Sn} 2-\mathrm{S} 7-\mathrm{Li} 3$ & $106.2(7)$ \\
\hline $\mathrm{S} 6-\mathrm{Sn} 2-\mathrm{S} 7$ & $111.30(6)$ & $\mathrm{Li} 2^{\mathrm{vii}}-\mathrm{S} 7-\mathrm{Li} 3$ & $108.1(10)$ \\
\hline $\mathrm{S} 5-\mathrm{Sn} 2-\mathrm{S} 4$ & $110.38(6)$ & $\mathrm{Sn} 2-\mathrm{S} 7-\mathrm{Cd}^{\mathrm{xii}}$ & $110.26(6)$ \\
\hline $\mathrm{S} 6-\mathrm{Sn} 2-\mathrm{S} 4$ & $105.19(5)$ & $\mathrm{Li} 2^{\mathrm{vii}}-\mathrm{S} 7-\mathrm{Cd}^{\mathrm{xii}}$ & $115.5(10)$ \\
\hline $\mathrm{S} 7-\mathrm{Sn} 2-\mathrm{S} 4$ & $107.74(7)$ & $\mathrm{Li} 3-\mathrm{S} 7-\mathrm{Cd}^{\mathrm{xii}}$ & $107.2(7)$ \\
\hline
\end{tabular}

Symmetry codes: (i) $x,-y+1, z-1 / 2$; (ii) $x,-y+2, z-1 / 2$; (iii) $x-1 / 2,-y+3 / 2, z-1 / 2$; (iv) $x-1 / 2, y+1 / 2, z$; (v) $x-1 / 2,-y+3 / 2, z+1 / 2$; (vi) $x-1 / 2, y-1 / 2, z$; (vii) $x,-y+2, z+1 / 2$; (viii) $x+1 / 2,-y+3 / 2, z+1 / 2$; (ix) $x+1 / 2, y-1 / 2, z$; (x) $x+1 / 2, y+1 / 2, z$; (xi) $x+1 / 2,-y+3 / 2, z-1 / 2$; (xii) $x,-y+1, z+1 / 2$. 
Table S6. Extended connectivity table for $\mathrm{Li}_{4} \mathrm{CdSn}_{2} \mathrm{~S}_{7}$ used to predict structural distortions according to Pauling's second rule. When the charge on the sulfur is compensated (CMP) by the cations in its first coordination sphere the coordination polyhedron is regular. When the charge of the sulfur is over CMP or under CMP cation-anion bonds will lengthen and shorten, respectively.

Horizontal

\begin{tabular}{|c|c|c|c|c|c|c|c|c|c|}
\hline & \multicolumn{7}{|c|}{ Anions } & \multirow{2}{*}{$\begin{array}{l}\text { Bond } \\
\text { Strength } \\
\text { Sums }\end{array}$} \\
\hline & & S1 & S2 & S3 & S4 & S5 & S6 & S7 & \\
\hline \multirow{8}{*}{ Cations } & Li1 & & 1 & 1 & 1 & 1 & & & \multirow[t]{2}{*}{$\Sigma=1$} \\
\hline & & & $\overline{4}$ & $\overline{4}$ & $\overline{4}$ & $\overline{4}$ & & & \\
\hline & Li2 & $\frac{1}{4}$ & & & & $\frac{1}{4}$ & $\frac{1}{4}$ & $\frac{1}{4}$ & $\Sigma=1$ \\
\hline & Li3 & $\frac{1}{4}$ & $\frac{1}{4}$ & $\frac{1}{4}$ & & & & $\frac{1}{4}$ & $\Sigma=1$ \\
\hline & Li4 & & & $\frac{1}{4}$ & $\frac{1}{4}$ & $\frac{1}{4}$ & $\frac{1}{4}$ & & $\Sigma=1$ \\
\hline & $\mathrm{Cd}$ & $\frac{2}{4}$ & $\frac{2}{4}$ & & & & $\frac{2}{4}$ & $\frac{2}{4}$ & $\Sigma=2$ \\
\hline & Sn1 & $\frac{4}{4}$ & $\frac{4}{4}$ & $\frac{4}{4}$ & $\frac{4}{4}$ & & & & $\Sigma=4$ \\
\hline & Sn2 & & & & $\frac{4}{4}$ & $\frac{4}{4}$ & $\frac{4}{4}$ & $\frac{4}{4}$ & $\Sigma=4$ \\
\hline \multicolumn{2}{|c|}{$\begin{array}{l}\text { Vertical Bond } \\
\text { Strength Sums }\end{array}$} & $\Sigma=2$ & $\Sigma=2$ & $\begin{array}{l}\Sigma=1.75 \\
1.75<2\end{array}$ & $\begin{array}{l}\Sigma=2.5 \\
2.5>2\end{array}$ & $\begin{array}{l}\Sigma=1.75 \\
1.75<2\end{array}$ & $\Sigma=2$ & $\Sigma=2$ & \\
\hline \multicolumn{2}{|c|}{$\begin{array}{r}\text { Charge } \\
\text { compensation }\end{array}$} & CMP & CMP & $\begin{array}{c}\text { UNDER } \\
\text { CMP }\end{array}$ & $\begin{array}{l}\text { OVER } \\
\text { CMP }\end{array}$ & $\begin{array}{c}\text { UNDER } \\
\text { CMP }\end{array}$ & CMP & CMP & \\
\hline
\end{tabular}


Table S7. Calculation of dipole moments, expressed in units of Debye (D), for each tetrahedron and net dipole moment for $\mathrm{Li}_{4} \mathrm{CdSn} \mathrm{S}_{2} \mathrm{~S}_{7}$.

\begin{tabular}{|c|c|c|c|c|}
\hline $\mathrm{Li}_{4} \mathrm{CdSn}{ }_{2} \mathrm{~S}_{7}(C c)$ & $x$-component (D) & $y$-component(D) & $z$-component(D) & Total moment(D) \\
\hline $\mathrm{Li}(1) \mathrm{S}_{4}$ & 0.040203015 & -0.248629004 & -0.014085175 & 0.25225197 \\
\hline $\mathrm{Li}(1) \mathrm{i}_{4}$ & 0.040203015 & -0.248709937 & -0.014085175 & 0.252331741 \\
\hline $\mathrm{Li}(1)^{\mathrm{ii}} \mathrm{S}_{4}$ & 0.040203015 & 0.248709937 & -0.014085175 & 0.252331741 \\
\hline $\mathrm{Li}(1)^{\mathrm{iii}} \mathrm{S}_{4}$ & 0.040203015 & 0.248709937 & -0.014085175 & 0.252331741 \\
\hline $\mathrm{Li}(2) \mathrm{S}_{4}$ & -0.055397343 & 0.098335149 & 0.449056936 & 0.463023541 \\
\hline $\mathrm{Li}(2)^{\prime} \mathrm{S}_{4}$ & -0.055397343 & 0.098335149 & 0.449056936 & 0.463023541 \\
\hline $\mathrm{Li}(2)^{\mathrm{iv}} \mathrm{S}_{4}$ & -0.055397343 & -0.098377564 & 0.449056936 & 0.463032551 \\
\hline $\mathrm{Li}(2)^{\mathrm{iv}} \mathrm{S}_{4}$ & -0.055397343 & -0.098377564 & 0.449056936 & 0.463032551 \\
\hline $\mathrm{Li}(3) \mathrm{S}_{4}$ & -0.004388135 & 0.187635434 & 0.233657424 & 0.299703359 \\
\hline $\mathrm{Li}(3)^{\mathrm{ivi}} \mathrm{S}_{4}$ & -0.004300829 & 0.187504475 & 0.233657424 & 0.299620121 \\
\hline $\mathrm{Li}(3)^{\mathrm{ii}} \mathrm{S}_{4}$ & -0.004388135 & -0.187635434 & 0.233657424 & 0.299703359 \\
\hline $\mathrm{Li}(3)^{\text {iviii }} \mathrm{S}_{4}$ & -0.004300829 & -0.187504475 & 0.233657424 & 0.299620121 \\
\hline $\mathrm{Li}(4) \mathrm{S}_{4}$ & -0.002093227 & 0.072255515 & 0.031178159 & 0.078723051 \\
\hline $\mathrm{Li}(4)^{\mathrm{ivi}} \mathrm{S}_{4}$ & -0.002093227 & 0.072255515 & 0.031178159 & 0.078723051 \\
\hline $\mathrm{Li}(4)^{\mathrm{ii}} \mathrm{S}_{4}$ & -0.002093227 & -0.072255515 & 0.031178159 & 0.078723051 \\
\hline $\mathrm{Li}(4)^{\text {iviii }} \mathrm{S}_{4}$ & -0.002093227 & -0.072255515 & 0.031178159 & 0.078723051 \\
\hline $\mathrm{Cd}(1)^{\mathrm{ii}} \mathrm{S}_{4}$ & 0.008173553 & 0.020359384 & 0.001948362 & 0.022045399 \\
\hline $\mathrm{Cd}(1)^{\mathrm{iviii}} \mathrm{S}_{4}$ & 0.00816733 & 0.020383773 & 0.001948362 & 0.022045399 \\
\hline $\mathrm{Cd}(1) \mathrm{S}_{4}$ & 0.008185548 & -0.020378501 & 0.001948362 & 0.022047281 \\
\hline $\mathrm{Cd}(1)^{\mathrm{ix}} \mathrm{S}_{4}$ & 0.008154585 & -0.02040289 & 0.001948362 & 0.022058361 \\
\hline $\mathrm{Sn}(1) \mathrm{S}_{4}$ & -0.064118792 & -0.32124343 & 0.128805473 & 0.351993481 \\
\hline $\mathrm{Sn}(1)^{\mathrm{i}} \mathrm{S}_{4}$ & -0.064118792 & -0.32124343 & 0.128805473 & 0.351993481 \\
\hline $\mathrm{Sn}(1)^{\mathrm{ii}} \mathrm{S}_{4}$ & -0.064118792 & 0.32124343 & 0.128805473 & 0.351993481 \\
\hline $\mathrm{Sn}(1)^{\mathrm{iii} S_{4}}$ & -0.064118792 & 0.32124343 & 0.128805473 & 0.351993481 \\
\hline $\mathrm{Sn}(2) \mathrm{S}_{4}$ & 0.076694647 & -0.066742723 & 0.220327748 & 0.242654026 \\
\hline $\mathrm{Sn}(2) \mathrm{S}_{4}$ & 0.076694647 & -0.066742723 & 0.220327748 & 0.242654026 \\
\hline $\operatorname{Sn}(2)^{i \mathrm{i}} \mathrm{S}_{4}$ & 0.076694647 & 0.066742723 & 0.220327748 & 0.242654026 \\
\hline $\mathrm{Sn}(2)^{\mathrm{iiii}} \mathrm{S}_{4}$ & 0.076694647 & 0.066742723 & 0.220327748 & 0.242654026 \\
\hline Sum per unit cell & -0.003543713 & -0.0000421303 & 4.203555713 & 4.203557207 \\
\hline$V\left(\AA^{3}\right)$ & \multicolumn{4}{|c|}{1246.39} \\
\hline $\begin{array}{l}\text { Dipole moment } \\
\left(D / \AA^{3}\right)\end{array}$ & \multicolumn{4}{|c|}{0.003372586} \\
\hline
\end{tabular}

Symmetry codes: (i) $0.5+x, 0.5+y, z$; (ii) $x, 1-y, 0.5+z$; (iii) $0.5+x, 0.5-y, 0.5+z$; (iv) $x, 1-y,-0.5+z$; (v) $0.5+x, 0.5-y,-0.5+z$; (vi) $\quad-0.5+x, 0.5+y, z$; (vii) $-0.5+x, 0.5-y, 0.5+z$; (viii) $0.5+x, 1.5-y, 0.5+z$; (ix) $0.5+x,-0.5+y, z$. 
Table S8. Calculation of dipole moments, expressed in units of Debye (D), for each tetrahedron and net dipole moment for $\mathrm{Li}_{2} \mathrm{CdSiS}_{4}$.

\begin{tabular}{|c|c|c|c|c|}
\hline $\mathrm{Li}_{2} \mathrm{CdSiS}_{4}\left(P m n 2_{1}\right)$ & $x$-component (D) & $y$-component(D) & $z$-component(D) & Total moment(D) \\
\hline $\mathrm{Li}(1) \mathrm{i}_{4}$ & 0.074099559 & 0.003169055 & -0.021492098 & 0.077218507 \\
\hline $\mathrm{Li}(1)^{\mathrm{ii}} \mathrm{S}_{4}$ & -0.074099559 & -0.003169055 & -0.021492098 & 0.077218507 \\
\hline $\mathrm{Li}(1)^{\mathrm{iii}} \mathrm{S}_{4}$ & 0.074099559 & -0.003169055 & -0.021492098 & 0.077218507 \\
\hline $\mathrm{Li}(1)^{\mathrm{iii}} \mathrm{S}_{4}$ & -0.074099559 & 0.003169055 & -0.021492098 & 0.077218507 \\
\hline $\mathrm{Cd}(1)^{\mathrm{iii}} \mathrm{S}_{4}$ & 0 & 0.07380448 & -0.015443798 & 0.075402999 \\
\hline $\mathrm{Cd}(1)^{\text {ivi }} \mathrm{S}_{4}$ & 0 & -0.07380448 & -0.015443798 & 0.075402998 \\
\hline $\mathrm{Si}(1)^{\text {iviii }} S_{4}$ & 0 & 0.226269617 & 0.055482908 & 0.23297273 \\
\hline $\mathrm{Si}(1)^{\text {iviiii }} S_{4}$ & 0 & -0.226269617 & 0.055482908 & 0.23297273 \\
\hline Sum per unit cell & 0 & 0 & -0.005890174 & 0.005890174 \\
\hline$V\left(\AA^{3}\right)$ & \multicolumn{4}{|c|}{325.9} \\
\hline $\begin{array}{l}\text { Dipole moment } \\
\left(\mathrm{D} / \AA^{3}\right)\end{array}$ & \multicolumn{4}{|c|}{0.0000180736} \\
\hline
\end{tabular}

Symmetry codes: (i) 0.5-x, 1-y, -0.5+z; (ii) 1+x, y, z; (iii) -x, y, z; (iv) 0.5+x, 1-y, -0.5+z; (v) 1+x, 1+y, z; (vi) $0.5-x,-y$, $-0.5+z$; (vii) $0.5-x, 1-y, 0.5+z ;$ (viii) $1+x, y, z$

Table S9. Calculation of dipole moments, expressed in units of Debye (D), for each tetrahedron and net dipole moment for $\mathrm{LiGaS}_{2}$.

\begin{tabular}{|c|c|c|c|c|}
\hline $\operatorname{LiGaS}_{2}\left(P_{n a 2}\right)$ & $x$-component (D) & $y$-component(D) & $z$-component(D) & Total moment(D) \\
\hline $\mathrm{Li}(1) \mathrm{S}_{4}$ & 0.147345809 & 0.027650352 & -0.132959663 & 0.200383636 \\
\hline $\mathrm{Li}(1)^{\mathrm{i}} \mathrm{S}_{4}$ & 0.147179334 & -0.027816827 & -0.132959663 & 0.200284309 \\
\hline $\mathrm{Li}(1)^{\mathrm{i}} \mathrm{S}_{4}$ & -0.147179334 & -0.027816827 & -0.132959663 & 0.200284309 \\
\hline $\mathrm{Li}(1)^{\mathrm{iii}} \mathrm{S}_{4}$ & -0.147179334 & 0.027816827 & -0.132959663 & 0.200284309 \\
\hline $\mathrm{Ga}(1) \mathrm{S}_{4}$ & -0.054850176 & 0.003438917 & -0.022638407 & 0.059437912 \\
\hline $\mathrm{Ga}(1)^{\mathrm{iv}} \mathrm{S}_{4}$ & -0.054651902 & -0.003438917 & -0.022638407 & 0.059254991 \\
\hline $\mathrm{Ga}(1)^{\mathrm{iv}} \mathrm{S}_{4}$ & 0.054651902 & 0.003438917 & -0.022638407 & 0.059254991 \\
\hline $\mathrm{Ga}(1)^{\mathrm{ivi}} \mathrm{S}_{4}$ & 0.054651902 & -0.003438917 & -0.022638407 & 0.059254991 \\
\hline Sum per unit cell & 0 & -0.000166475 & -0.622392279 & 0.622392302 \\
\hline$V\left(\AA^{3}\right)$ & \multicolumn{4}{|c|}{318.42} \\
\hline $\begin{array}{l}\text { Dipole moment } \\
\left(D / \AA^{3}\right)\end{array}$ & \multicolumn{4}{|c|}{0.001954627} \\
\hline
\end{tabular}

Symmetry codes: (i) $0.5+x, 1.5-y, z$; (ii) $1-x, 1-y, 0.5+z$; (iii) $0.5-x,-0.5+y, 0.5+z$; (iv) $0.5+x, 1.5-y, z$; (v) $0.5-x,-0.5+y,-$ $0.5+z$; (vi) $1-x, 1-y,-0.5+z$. 
Table S10. Calculation of dipole moments, expressed in units of Debye (D), for each tetrahedron and net dipole moment for LilnS 2 .

\begin{tabular}{|c|c|c|c|c|}
\hline $\operatorname{LilnS}_{2}\left(P 2_{1} n b\right)$ & $x$-component (D) & $y$-component(D) & $z$-component(D) & Total moment(D) \\
\hline $\mathrm{Li}(1) \mathrm{S}_{4}$ & 0.249332531 & 0.114173196 & 0.223847612 & 0.353991502 \\
\hline $\mathrm{Li}(1) \mathrm{S}_{4}$ & 0.249332531 & 0.114173196 & -0.223847612 & 0.353991502 \\
\hline $\mathrm{Li}(1)^{\mathrm{ii}} \mathrm{S}_{4}$ & 0.249209492 & -0.114296235 & -0.223847612 & 0.353944563 \\
\hline $\mathrm{Li}(1)^{\mathrm{iii}} \mathrm{S}_{4}$ & 0.249209492 & -0.114296235 & 0.223847612 & 0.353944563 \\
\hline $\ln (1) S_{4}$ & 0.051695461 & 0.009514243 & -0.04340551 & 0.06816876 \\
\hline $\ln (1)^{\mathrm{iix}} S_{4}$ & 0.051695461 & -0.009514243 & -0.04340551 & 0.06816876 \\
\hline $\ln (1)^{i x} S_{4}$ & 0.051695461 & -0.009514243 & 0.04340551 & 0.06816876 \\
\hline $\ln (1)^{\prime} S_{4}$ & 0.051695461 & 0.009514243 & 0.04340551 & 0.06816876 \\
\hline Sum per unit cell & 1.203865891 & -0.000246077 & 0 & 1.203865916 \\
\hline$V\left(\AA^{3}\right)$ & \multicolumn{4}{|c|}{358.92} \\
\hline $\begin{array}{l}\text { Dipole moment } \\
\left(D / \AA^{3}\right)\end{array}$ & \multicolumn{4}{|c|}{0.003354134} \\
\hline
\end{tabular}

Symmetry codes: (i) $x, 0.5+y, 0.5-z$; (ii) $-0.5+x, 1-y, 1-z$; (iii) $-0.5+x, 0.5-y, 0.5+z$; (iv) $0.5+x, 0.5-y, 0.5+z$; (v) $0.5+x, 1-y$, $1-z$.

Table S11. Calculation of dipole moments, expressed in units of Debye (D), for each tetrahedron and net dipole moment for LilnSe $e_{2}$.

\begin{tabular}{|c|c|c|c|c|}
\hline $\operatorname{LilnSe}_{2}\left(P_{n a 2_{1}}\right)$ & $x$-component (D) & $y$-component(D) & $z$-component(D) & Total moment(D) \\
\hline $\mathrm{Li}(1) \mathrm{Se}_{4}$ & -0.078174838 & 0.035122543 & -0.070863371 & 0.111204837 \\
\hline $\mathrm{Li}(1) \mathrm{Se}_{4}$ & -0.078174838 & -0.035122543 & -0.070863371 & 0.111204837 \\
\hline $\mathrm{Li}(1)^{\mathrm{ii}} \mathrm{Se}_{4}$ & 0.078212554 & 0.035122543 & -0.070863371 & 0.111231354 \\
\hline $\mathrm{Li}(1)^{\mathrm{iii}} \mathrm{Se}_{4}$ & 0.078212554 & -0.035122543 & -0.070863371 & 0.111231354 \\
\hline $\ln (1) \mathrm{Se}_{4}$ & -2.871681515 & 1.128861817 & 0.421655863 & 3.114269961 \\
\hline $\ln (1)^{i} \mathrm{Se}_{4}$ & -2.870965611 & -1.128861817 & 0.421655863 & 3.113609835 \\
\hline $\ln (1)^{\mathrm{iix}} \mathrm{Se}_{4}$ & 2.870965611 & 1.128861817 & 0.421655863 & 3.113609835 \\
\hline $\ln (1)^{\mathrm{ix}} \mathrm{Se}_{4}$ & 2.871681515 & -1.128861817 & 0.421655863 & 3.114269961 \\
\hline Sum per unit cell & 0 & 0 & 1.403169966 & 1.403169966 \\
\hline$V\left(\AA^{3}\right)$ & \multicolumn{4}{|c|}{413.42} \\
\hline $\begin{array}{l}\text { Dipole moment } \\
\left(D / \AA^{3}\right)\end{array}$ & \multicolumn{4}{|c|}{0.003394} \\
\hline
\end{tabular}

Symmetry codes: (i) $0.5+x, 0.5-y, z$; (ii) $0.5-x, 0.5+y, 0.5+z$; (iii) 1-x, 1-y, 0.5+z; (iv) 0.5-x, 0.5+y, -0.5+z; (v) 1-x, 1-y, $0.5+z$. 
Table S12. The calculated Mulliken bond populations for $\mathrm{Li}_{4} \mathrm{CdSn}_{2} \mathrm{~S}_{7}$.

\begin{tabular}{|l|l|l||l|l|l|}
\hline bond & length $(\AA)$ & population & bond & Length $(\AA)$ & population \\
\hline Li1-S5 & 2.38822 & -0.04 & Li1-S2 & 2.43081 & -0.12 \\
\hline Li1-S3 & 2.41683 & -0.02 & Li1-S4 & 2.53584 & -0.11 \\
\hline Li2-S6 & 2.25804 & -0.04 & Li2-S1 & 2.56664 & -0.07 \\
\hline Li2-S5 & 2.54632 & -0.08 & Li2-S7 & 2.40604 & -0.13 \\
\hline Li3-S1 & 2.26480 & -0.11 & Li3-S3 & 2.49291 & 0.04 \\
\hline Li3-S2 & 2.42640 & -0.11 & Li3-S7 & 2.51552 & -0.02 \\
\hline Li4-S5 & 2.41866 & 0.02 & Li4-S3 & 2.43911 & -0.01 \\
\hline Li4-S6 & 2.43969 & -0.10 & Li4-S4 & 2.49041 & -0.14 \\
\hline Cd1-S2 & 2.53179 & 0.54 & Cd1-S1 & 2.53422 & 0.48 \\
\hline Cd1-S6 & 2.53605 & 0.42 & Cd1-S7 & 2.53883 & 0.47 \\
\hline Sn1-S3 & 2.34349 & 0.94 & Sn1-S2 & 2.38165 & 0.68 \\
\hline Sn1-S1 & 2.38704 & 0.71 & Sn1-S4 & 2.46144 & 0.55 \\
\hline Sn2-S5 & 2.34266 & 0.82 & Sn2-S6 & 2.37520 & 0.74 \\
\hline Sn2-S7 & 2.37866 & 0.72 & Sn2-S4 & 2.45789 & 0.54 \\
\hline
\end{tabular}


Table S12. Chalcogenides for which both second harmonic generation and laser-induced damage threshold (LIDT) have been reported. Compounds that are phase matchable (PM) with SHG responses comparable to or greater than AGS and a LIDT $>8 \times$ AGS are highlight in blue. The title compound, $\mathrm{Li}_{4} \mathrm{CdSn}_{2} \mathrm{~S}_{7}$ is highlight in yellow.

\begin{tabular}{|c|c|c|c|c|c|c|c|c|c|c|}
\hline Compound & $\begin{array}{l}\mathrm{Eg} \\
(\mathrm{eV})\end{array}$ & $\begin{array}{c}\text { LIDT } \\
\left(\mathrm{MW} / \mathrm{cm}^{2}\right)\end{array}$ & $\begin{array}{c}\text { LIDT } \\
\text { (x reference) }\end{array}$ & $\begin{array}{l}\lambda \text { for } \\
\text { LIDT } \\
(\mu \mathrm{m})\end{array}$ & $\begin{array}{l}\tau \text { for } \\
\text { LIDT }\end{array}$ & $\begin{array}{l}\text { Spot size } \\
(\mathrm{mm})\end{array}$ & SHG & PM or NPM & $\begin{array}{c}\text { Reference } \\
\text { Quality }\end{array}$ & Ref \\
\hline $\mathrm{Li}\left[\mathrm{LiCs}_{2} \mathrm{Cl}\right]\left[\mathrm{Ga}_{3} \mathrm{~S}_{6}\right]$ & 4.18 & 123 & 4.1x AGS & 1.064 & $10 \mathrm{~ns}$ & NS & 0.7x AGS @1.91 um & PM @1.91 $\mu \mathrm{m}$ & NS & 2 \\
\hline$\alpha-\mathrm{Li}_{2} \mathrm{ZnGeS}_{4}$ & 4.07 & 12,300 & $41 \times$ AGS & 1.064 & $30 \mathrm{ps}$ & 0.5 & $\chi^{(2)}=26 \mathrm{pm} / \mathrm{V}^{\mathrm{SL}}$ & PM @3.1 $\mu \mathrm{m}$ & $\mathrm{OQ}$ & 3 \\
\hline $\mathrm{SrLi}_{2} \mathrm{SiS}_{4}$ & 3.94 & NS & 21s AGS & 1.06 & NS & NS & $0.4 x$ AGS @2.09 $\mu \mathrm{m}$ & PM @ $2.09 \mu \mathrm{m}$ & NS & 4 \\
\hline $\mathrm{SrNa}_{2} \mathrm{SiS}_{4}$ & 3.87 & NS & $17 \mathrm{~s}$ AGS & 1.06 & NS & NS & $0.4 x$ AGS @2.09 $\mu \mathrm{m}$ & PM @ $2.09 \mu \mathrm{m}$ & NS & 4 \\
\hline $\mathrm{Li}_{2} \mathrm{ZnSiS}_{4}$ & 3.9 & NS & 10x AGS & 1.064 & NS & NS & 1.1xAGS@2.09 um & PM @2.09 $\mu \mathrm{m}$ & NS & 5 \\
\hline$L_{i G a} 0.54 \mathrm{In}_{0.46} \mathrm{~S}_{2}$ & 3.86 & 35.7 & $12 \times A G S$ & 1.064 & NS & NS & 0.6x AGS @1.91 $\mu \mathrm{m}$ & $\mathrm{NPM} @ 1.91 \mu \mathrm{m}$ & NS & 6 \\
\hline \multirow[t]{2}{*}{$\mathrm{Li}_{2} \mathrm{CdSiS}_{4}$} & 3.76 & NM & NM & NM & NM & NS & $\sim A G S$ & PM @2.09 $\mu \mathrm{m}$ & NS & 7 \\
\hline & 4.02 & $>2,500$ & $>8.3 \times$ AGS & 1.064 & $30 \mathrm{ps}$ & 0.5 & $\chi^{(2)}=14.8 \mathrm{pm} / \mathrm{V}^{\mathrm{SL}}$ & PM @3.1 $\mu \mathrm{m}$ & $\mathrm{OQ}$ & This work \\
\hline $\mathrm{Na}_{2} \mathrm{BaGeS}_{4}$ & 3.70 & 43.5 & $8 \times$ AGS & 1.06 & $10 \mathrm{~ns}$ & NS & $0.3 x$ AGS @2.09 $\mu \mathrm{m}$ & PM @2.09 $\mu \mathrm{m}$ & NS & 8 \\
\hline $\mathrm{Li}_{2} \mathrm{BaGeS}_{4}$ & 3.66 & 325 & $11 \times A G S$ & 1.064 & $10 \mathrm{~ns}$ & NS & $0.5 x$ AGS @2.09 $\mu \mathrm{m}$ & PM @2.09 $\mu \mathrm{m}$ & NS & 9 \\
\hline $\mathrm{Rb}_{10} \mathrm{Zn}_{4} \mathrm{Sn}_{4} \mathrm{~S}_{17}$ & 3.59 & 157 & $5 \times$ AGS & 1.06 & $10 \mathrm{~ns}$ & NS & 0.7x AGS @1.06 $\mu \mathrm{m}$ & NPM @1.06 $\mu \mathrm{m}$ & NS & 10 \\
\hline $\mathrm{BaGa}_{4} \mathrm{~S}_{7}$ & 3.54 & $12.1^{\#}$ & NS & 1.064 & $15 \mathrm{~ns}$ & NS & 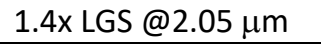 & 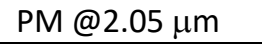 & NS & 11 \\
\hline $\mathrm{Ba}_{6} \mathrm{Zn}_{7} \mathrm{Ga}_{2} \mathrm{~S}_{16}$ & 3.50 & 40.47 & 28xAGS & 1.064 & $10 \mathrm{~ns}$ & NS & $0.5 \times A G S$ & PM@2.05 $\mu \mathrm{m}$ & NS & 12 \\
\hline$\beta-\mathrm{Li}_{2} \mathrm{ZnGeS}{ }_{4}$ & 3.49 & 242 & 8x AGS & 1.064 & $10 \mathrm{~ns}$ & NS & $0.7 x$ AGS @2.09 $\mu \mathrm{m}$ & 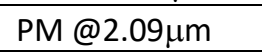 & NS & 13 \\
\hline $\mathrm{LiBa}_{4} \mathrm{Ga}_{5} \mathrm{~S}_{12}$ & 3.43 & 190 & $21 \times A G S$ & 1.064 & $10 \mathrm{~ns}$ & NS & 1.5x AGS @2.09 $\mu \mathrm{m}^{\%}$ & NPM @2.09 $\mu \mathrm{m}$ & NS & 14 \\
\hline $\mathrm{Ba}_{13} \mathrm{Zn}_{7} \mathrm{In}_{12} \mathrm{~S}_{38}$ & 3.35 & 68 & $24 x \mathrm{AGS}$ & 1.064 & $10 \mathrm{~ns}$ & NS & 0.6x AGS @1.91 rm & PM @1.91 $\mu \mathrm{m}$ & NS & 15 \\
\hline$\left[\mathrm{CsBa}_{2} \mathrm{Cl}\right]\left[\mathrm{Ga}_{4} \mathrm{~S}_{8}\right]$ & 3.35 & 90.4 & $12 \times \mathrm{AGS}$ & 1.064 & $10 \mathrm{~ns}$ & NS & $0.9 x$ AGS @1.91 $\mu \mathrm{m}$ & PM @1.91 $\mu \mathrm{m}$ & NS & 16 \\
\hline $\mathrm{Rb}_{2} \mathrm{Ga}_{2} \mathrm{P}_{2} \mathrm{~S}_{9}$ & 3.30 & NS & 7x AGS & 1.064 & $10 \mathrm{~ns}$ & NS & $0.1 \times$ AGS $2.05 \mu \mathrm{m}$ & PM @1.064 & NS & 17 \\
\hline$\left[\mathrm{RbBa}_{2} \mathrm{Cl}\right]\left[\mathrm{Ga}_{4} \mathrm{~S}_{8}\right]$ & 3.30 & 82.4 & $11 \times A G S$ & 1.064 & 10ns & NS & 1x AGS @1.91 um & PM@1.91 $\mu \mathrm{m}$ & NS & 16 \\
\hline $\mathrm{Na}_{2} \mathrm{BaSnS}_{4}$ & 3.27 & 27.8 & $5 \times A G S$ & 1.06 & $10 \mathrm{~ns}$ & NS & $0.5 x$ AGS @2.09 $\mu \mathrm{m}$ & PM@2.09 $\mu \mathrm{m}$ & NS & 8 \\
\hline $\mathrm{Na}_{2} \mathrm{ZnGe}_{2} \mathrm{~S}_{6}$ & 3.25 & 228 & 6x AGS & 1.064 & $10 \mathrm{~ns}$ & NS & 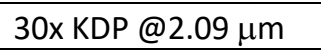 & PM @2.09 $\mu \mathrm{m}$ & $\mathrm{NS}^{* *}$ & 18 \\
\hline $\mathrm{Li}_{2} \mathrm{CdGeS}_{4}$ & 3.15 & 4,000 & 13.3x AGS & 1.064 & $30 \mathrm{ps}$ & 0.5 & $\chi^{(2)}=22.5 \mathrm{pm} / \mathrm{V}^{\mathrm{SL}}$ & $\mathrm{PM} \geq 1.5 \mu \mathrm{m}$ & $\mathrm{OQ}$ & 19 \\
\hline $\mathrm{Cu}_{2} \mathrm{ZnSiS}_{4}$ & $\sim 3.1$ & 2,000 & $6.7 x$ AGS & 1.064 & $30 \mathrm{ps}$ & 0.5 & $\chi^{(2)}=6.6 \mathrm{pm} / \mathrm{V}^{\mathrm{SL}}$ & $\mathrm{PM} \geq 1.7 \mu \mathrm{m}$ & $\mathrm{OQ}$ & 20 \\
\hline $\mathrm{Na}_{2} \mathrm{Ga}_{2} \mathrm{GeS}_{6}$ & 3.10 & 103.55 & $18.1 \times$ AGS & 1.064 & $10 \mathrm{~ns}$ & $0.0616 \mathrm{~cm}^{3^{*}}$ & $0.8 x$ AGS @1.91 $\mu \mathrm{m}$ & PM@ $1.91 \mu \mathrm{m}$ & NS & 21 \\
\hline $\mathrm{SnGa}_{4} \mathrm{~S}_{7}$ & 3.10 & 165.1 & 19x AGS & 1.064 & $8 \mathrm{~ns}$ & 3.1 & 1.3x AGS @2.05 $\mu \mathrm{m}$ & PM@2.05 $\mu \mathrm{m}$ & NS & 22 \\
\hline $\mathrm{Li}_{2} \mathrm{BaSnS}_{4}$ & 3.07 & 192 & $6.5 \times$ AGS & 1.064 & $10 \mathrm{~ns}$ & NS & $0.7 x$ AGS @2.09 $\mu \mathrm{m}$ & 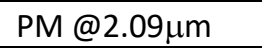 & NS & 9 \\
\hline $\mathrm{Li}_{2} \mathrm{MnGeS}_{4}$ & 3.07 & $>16,000$ & $>53 \times$ AGS & 1.064 & $30 \mathrm{ps}$ & 0.5 & $\chi^{(2)}=6.6 \mathrm{pm} / \mathrm{V}^{\mathrm{SL}}$ & $\mathrm{PM} \geq 1.6 \mu \mathrm{m}$ & $\mathrm{OQ}$ & 23 \\
\hline $\mathrm{SrCu}_{2} \mathrm{SiS}_{4}$ & 3.04 & $\mathrm{NS}$ & 8x AGS & 1.06 & NS & NS & $0.7 x$ AGS @2.09 $\mu \mathrm{m}$ & PM @ $2.09 \mu \mathrm{m}$ & NS & 4 \\
\hline
\end{tabular}




\begin{tabular}{|c|c|c|c|c|c|c|c|c|c|c|}
\hline $\mathrm{Sr}_{3} \mathrm{MnSn}_{2} \mathrm{~S}_{8}$ & 3.02 & NS & $3 \times A G S$ & 1.06 & NS & NS & AGS @1.06 $\mu \mathrm{m}$ & NPM & NS & 24 \\
\hline $\mathrm{Ba}_{2} \mathrm{Ga}_{8} \mathrm{GeS}_{16}$ & 3.0 & 83.8 & $22 x$ AGS & 1.064 & $8 \mathrm{~ns}$ & 1.9 & $\begin{array}{l}\chi^{(2)}=6.6 \mathrm{pm} / \mathrm{V} \\
@ 1.91 \mu \mathrm{m}\end{array}$ & 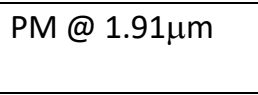 & NS & 25 \\
\hline $\mathrm{Ba}_{10} \mathrm{Zn}_{7} \mathrm{In}_{6} \mathrm{~S}_{26}$ & 3.0 & 53.54 & $37.2 \times$ AGS & 1.064 & $10 \mathrm{~ns}$ & NS & 4.1x AGS@2.05 $\mu \mathrm{m}$ & NPM @ $2.05 \mu \mathrm{m}$ & $x$ & 26 \\
\hline $\mathrm{CuZnPS}_{4}$ & 3.0 & 35.83 & 6x AGS & 1.064 & $8 \mathrm{~ns}$ & NS & 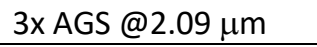 & PM @ $2.09 \mu \mathrm{m}$ & NS & 27 \\
\hline $\mathrm{Ba}_{10} \mathrm{In}_{6} \mathrm{Zn}_{7} \mathrm{~S}_{26}$ & 2.95 & NS & 13.7x AGS & 1.064 & $10 \mathrm{~ns}$ & NS & $0.5 x$ AGS @2.05 $\mu \mathrm{m}$ & NS & NS & 28 \\
\hline $\mathrm{Li}_{2} \mathrm{Ba}_{6} \mathrm{MnSn}_{4} \mathrm{~S}_{16}$ & 2.88 & NM & NM & NM & NS & NS & 5.1x AGS @2.05 $\mu \mathrm{m}$ & NPM @2.05 $\mu \mathrm{m}$ & NS & 29 \\
\hline $\mathrm{Dy}_{3} \mathrm{GaS}_{6}$ & 2.88 & 79.45 & $14 \times$ AGS & 1.064 & $8 \mathrm{~ns}$ & 2 & 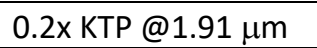 & PM @ $1.91 \mu \mathrm{m}$ & NS & 30 \\
\hline $\mathrm{Na}_{2} \mathrm{Hg}_{3} \mathrm{Si}_{2} \mathrm{~S}_{8}$ & 2.86 & 54 & 4.5x AGS & 1.06 & $10 \mathrm{~ns}$ & NS & 1.3 x AGS @2.09 $\mu \mathrm{m}$ & PM @2.09 $\mu \mathrm{m}$ & NS & 31 \\
\hline $\mathrm{Na}_{4} \mathrm{MgSi}_{2} \mathrm{Se}_{6}$ & 2.85 & 45.8 & 9x AGS & 1.06 & $10 \mathrm{~ns}$ & NS & $0.5 x$ AGS @2.09 $\mu \mathrm{m}$ & NM & NS & 32 \\
\hline $\mathrm{LiSm}_{3} \mathrm{SiS}_{7}$ & 2.83 & 118 & $3.7 \times$ AGS & 1.064 & $10 \mathrm{~ns}$ & 0.25 & 1.5x AGS@2.09 $\mu \mathrm{m}$ & NPM $2.09 \mu \mathrm{m}$ & NS & 33 \\
\hline $\mathrm{Y}_{3} \mathrm{GaS}_{6}$ & 2.81 & 103.78 & $18 \times A G S$ & 1.064 & $8 \mathrm{~ns}$ & 2 & 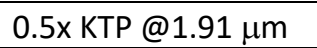 & PM @1.91 $\mu \mathrm{m}$ & NS & 30 \\
\hline $\mathrm{Sr}_{6} \mathrm{Ag}_{2} \mathrm{Cu}_{2} \mathrm{Ge}_{4} \mathrm{~S}_{16}$ & 2.76 & NS & $4.5 \times A G S$ & 1.06 & NS & NS & 1.1x AGS @2.09 $\mu \mathrm{m}$ & NPM & NS & 34 \\
\hline $\mathrm{Ag}_{2} \mathrm{Ba}_{6} \mathrm{MnSn}_{4} \mathrm{~S}_{16}$ & 2.76 & NM & NM & NS & NS & NS & 2.7x AGS@2.05 $\mu \mathrm{m}$ & NPM @2.05 m & NS & 29 \\
\hline $\mathrm{Cd}_{4} \mathrm{SiS}_{6}$ & 2.75 & 18.9 & $8.6 x$ AGS & 1.064 & $10 \mathrm{~ns}$ & NS & 1.3x AGS @2.05 $\mu \mathrm{m}$ & NPM@2.05 $\mu \mathrm{m}$ & NS & 35 \\
\hline $\mathrm{AgGa}_{2} \mathrm{PS}_{6}$ & 2.75 & NS & $5.1 \times A G S$ & 1.064 & $10 \mathrm{~ns}$ & 5.8 & 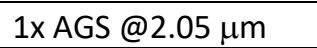 & PM @2.05 $\mu \mathrm{m}$ & NS & 36 \\
\hline $\mathrm{Na}_{2} \mathrm{Ga}_{2} \mathrm{SnS}_{6}$ & 2.74 & 102.87 & 17.9x AGS & 1.064 & $10 \mathrm{~ns}$ & $0.0346 \mathrm{~cm}^{3^{*}}$ & 1.1x AGS@1.91 $\mu \mathrm{m}$ & PM@1.91 $\mu \mathrm{m}$ & NS & 21 \\
\hline $\mathrm{Na}_{2} \mathrm{Hg}_{3} \mathrm{Ge}_{2} \mathrm{~S}_{8}$ & 2.68 & 36 & $3 x$ AGS & 1.06 & $10 \mathrm{~ns}$ & NS & 2.2 x AGS@2.09 $\mu \mathrm{m}$ & PM @2.09 $\mu \mathrm{m}$ & NS & 31 \\
\hline $\mathrm{Ba}_{10} \mathrm{In}_{6} \mathrm{Zn}_{7} \mathrm{~S}_{10} \mathrm{Se}_{16}$ & 2.65 & NS & 12.3x AGS & 1.064 & $10 \mathrm{~ns}$ & NS & 0.7x AGS @2.05 ㅆm & PM@2.05 $\mu \mathrm{m}$ & NS & 28 \\
\hline $\mathrm{BaGa}_{4} \mathrm{Se}_{7}$ & 2.64 & 557 & 3.7x AGS & 1.064 & $5 \mathrm{~ns}$ & 0.4 & 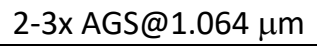 & $\mathrm{PM}>0.47 \mu \mathrm{m}$ & $\mathrm{SC}$ & 37 \\
\hline $\mathrm{Sr}_{6} \mathrm{Ag}_{4} \mathrm{Ge}_{4} \mathrm{~S}_{16}$ & 2.62 & NS & $4.5 \times A G S$ & 1.06 & NS & NS & 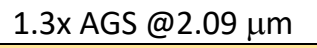 & NPM & NS & 34 \\
\hline $\mathrm{Li}_{4} \mathrm{CdSn} \mathrm{S}_{7}$ & 2.59 & $>2,500$ & $>8.3 \times$ AGS & 1.064 & $30 \mathrm{ps}$ & 0.5 & $\chi^{(2)}=35.0 \mathrm{pm} / \mathrm{V}^{S L}$ & PM @3.1 $\mu \mathrm{m}$ & $\mathrm{OQ}$ & This work \\
\hline $\mathrm{BaCdGeS}_{4}$ & 2.58 & 39.725 & 12xAGS & 1.064 & $10 \mathrm{~ns}$ & $0.3 \mathrm{~cm}^{2 *}$ & 0.3xAGS @ $1.91 \mu \mathrm{m}$ & PM @ $1.9 \mu \mathrm{m}$ & NS & 38 \\
\hline $\mathrm{SnGa}_{4} \mathrm{Se}_{7}$ & 2.55 & 40.0 & 4.6x AGS & 1.064 & $8 \mathrm{~ns}$ & 5.2 & 3.8x AGS@2.05 $\mu \mathrm{m}$ & PM @2.05 $\mu \mathrm{m}$ & NS & 22 \\
\hline $\mathrm{Na}_{4} \mathrm{MgGe}_{2} \mathrm{Se}_{6}$ & 2.53 & 35.8 & 7x AGS & 1.06 & $10 \mathrm{~ns}$ & NS & 1.3x AGS@2.09 $\mu \mathrm{m}$ & PM @2.09 $\mu \mathrm{m}$ & NS & 32 \\
\hline $\mathrm{Cd}_{4} \mathrm{GeS}_{6}$ & 2.52 & NS & 3.6x AGS & 1.064 & $8 \mathrm{~ns}$ & NS & 1.1s AGS@2.05 m & $\mathrm{PM} @ 2.05 \mu \mathrm{m}$ & NS & 39 \\
\hline $\mathrm{Li}_{2} \mathrm{CdGeSe}_{4}$ & 2.5 & 300 & $\sim A G S$ & 1.064 & $30 \mathrm{ps}$ & 0.5 & $\chi^{(2)}=25.6 \mathrm{pm} / \mathrm{V}^{\mathrm{SL}}$ & $\mathrm{PM} \geq 2.7 \mu \mathrm{m}$ & $\mathrm{OQ}$ & 40 \\
\hline $\mathrm{Li}_{2} \mathrm{ZnGeSe}{ }_{4}$ & 2.5 & 300 & $\sim$ AGS & 1.064 & $30 \mathrm{ps}$ & 0.5 & $\chi^{(2)}=19 \mathrm{pm} / \mathrm{V}^{\mathrm{SL}}$ & $\mathrm{PM} \geq 2.5 \mu \mathrm{m}$ & $\mathrm{OQ}$ & \\
\hline $\mathrm{Na}_{2} \mathrm{Hg}_{3} \mathrm{Sn}_{2} \mathrm{~S}_{8}$ & 2.45 & 12 & $\sim A G S$ & 1.06 & $10 \mathrm{~ns}$ & NS & 2.8 x AGS @2.09 $\mu \mathrm{m}$ & PM @2.09 $\mu \mathrm{m}$ & NS & 31 \\
\hline $\mathrm{LiBa}_{4} \mathrm{Ga}_{5} \mathrm{Se}_{12}$ & 2.44 & 56 & 6x AGS & 1.064 & $10 \mathrm{~ns}$ & NS & 1.5x AGS @2.09 $\mu \mathrm{m}^{\%}$ & NPM @2.09 $\mu \mathrm{m}$ & NS & 14 \\
\hline $\mathrm{Li}_{2} \mathrm{BaGeSe}_{4}$ & 2.40 & 29.6 & $\sim A G S$ & 1.064 & $10 \mathrm{~ns}$ & NS & 1.1x AGS@2.09 $\mu \mathrm{m}$ & PM @2.09 $\mu \mathrm{m}$ & NS & 9 \\
\hline $\mathrm{Ba}_{2} \mathrm{SnS}_{5}$ & 2.38 & 18.2 & 7.9x AGS & 1.064 & $10 \mathrm{~ns}$ & 3.74 & 1.1x AGS@1.57 $\mu \mathrm{m}$ & $\mathrm{PM} @ 1.57 \mu \mathrm{m}$ & NS & 42 \\
\hline $\mathrm{Sr}_{2} \mathrm{SnS}_{5}$ & 2.38 & 16.4 & 7.1x AGS & 1.064 & $10 \mathrm{nx}$ & 3.74 & 0.3x AGS@1.57 $\mu \mathrm{m}$ & PM @1.57 $\mu \mathrm{m}$ & NS & 42 \\
\hline $\mathrm{Ba}_{10} \mathrm{In}_{6} \mathrm{Zn}_{7} \mathrm{Se}_{26}$ & 2.28 & NS & 10x AGS & 1.064 & $10 \mathrm{~ns}$ & NS & 0.8x AGS@2.05 $\mu \mathrm{m}$ & PM @2.05 $\mu \mathrm{m}$ & NS & 28 \\
\hline $\mathrm{Li}_{2} \mathrm{CdSnSe}{ }_{4}$ & 2.2 & 700 & $2.3 \times$ AGS & 1.064 & $30 \mathrm{ps}$ & 0.5 & $\chi^{(2)}=25.3 \mathrm{pm} / \mathrm{V}^{\mathrm{SL}}$ & $\mathrm{PM} \geq 2.9 \mu \mathrm{m}$ & $\mathrm{OQ}$ & 40 \\
\hline
\end{tabular}




\begin{tabular}{|c|c|c|c|c|c|c|c|c|c|c|}
\hline $\mathrm{Ba}_{6} \mathrm{In}_{6} \mathrm{Zn}_{4} \mathrm{Se}_{19}$ & 2.2 & 34.6 & $15.5 \times A G S$ & 1.064 & $10 \mathrm{~ns}$ & NS & 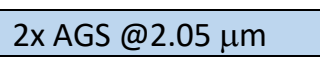 & PM@2.05 $\mu \mathrm{m}$ & NS & 43 \\
\hline $\mathrm{Li}_{2} \mathrm{BaSnSe}_{4}$ & 2.18 & 29.6 & $\sim A G S$ & 1.064 & $10 \mathrm{~ns}$ & NS & 1.3x AGS@2.09 $\mu \mathrm{m}$ & 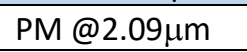 & NS & 9 \\
\hline $\mathrm{NaIn}_{3} \mathrm{Se}_{5}$ & 2.17 & 12.8 & 10.7x AGS & 1.064 & $10 \mathrm{~ns}$ & 6.2 & 0.3 x AGS @1.91 $\mu \mathrm{m}$ & 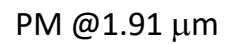 & NS & 44 \\
\hline $\mathrm{Ba}_{12} \mathrm{Zn}_{8} \ln _{12} \mathrm{~S}_{38}$ & 2.15 & 46 & $16 x$ AGS & 1.064 & $10 \mathrm{~ns}$ & NS & $0.16 x$ AGS @1.91 $\mu \mathrm{m}$ & NPM @1.91 $\mu \mathrm{m}$ & NS & 15 \\
\hline $\mathrm{Rb}_{2} \mathrm{Ag}_{3} \mathrm{Sb}_{3} \mathrm{~S}_{7}$ & 2.11 & 3.53 & $2.3 \times A G S$ & 1.064 & $10 \mathrm{~ns}$ & NS & $0.5 x$ AGS @2.1 $\mu \mathrm{m}$ & PM @2.1 $\mu \mathrm{m}$ & NS & 45 \\
\hline$\delta-\mathrm{Ga}_{2} \mathrm{Se}_{3}$ & 2.10 & 39.4 & 16.7x AGS & 1.064 & $10 \mathrm{~ns}$ & NS & 2.3x AGS @1.91 $\mu \mathrm{m}$ & PM & NS & 46 \\
\hline $\mathrm{KInSi}_{1.32} \mathrm{Sn}_{0.68} \mathrm{Se}_{6}$ & 2.10 & 2.07 & $0.8 \times$ AGS & 1.064 & $10 \mathrm{~ns}$ & NS & 1.3x AGS @2.1 $\mu \mathrm{m}$ & PM @2.1 $\mu \mathrm{m}$ & NS & 47 \\
\hline $\mathrm{Sr}_{6} \mathrm{Ag}_{4} \mathrm{Sn}_{4} \mathrm{~S}_{16}$ & 2.07 & NS & 2.0xAGS & 1.06 & NS & NS & 2.0x AGS @2.09 $\mu \mathrm{m}$ & NPM & NS & 34 \\
\hline$\alpha-\mathrm{SrCu}_{2} \mathrm{SnS}_{4}$ & 2.06 & NS & $2.3 \times$ AGS & 1.06 & $50 \mathrm{~ns}$ & NS & $0.7 x$ AGS @2.09 $\mu \mathrm{m}$ & PM @2.09 $\mu \mathrm{m}$ & NS & 48 \\
\hline $\mathrm{SrCdSnS}_{4}$ & 2.05 & 31.067 & $10 \times \mathrm{AGS}$ & 1.064 & $10 \mathrm{~ns}$ & $0.196 \mathrm{~cm}^{2^{*}}$ & 1.3x AGS @2.04 $\mu \mathrm{m}$ & PM @2.04 $\mu \mathrm{m}$ & NS & 49 \\
\hline $\mathrm{HgCuPS}_{4}$ & 2.03 & 6.05 & $4.2 \times A G S$ & 1.064 & $10 \mathrm{~ns}$ & NS & $6.5 x$ AGS @2.05 $\mu \mathrm{m}$ & PM @2.05 $\mu \mathrm{m}$ & NS & 50 \\
\hline$\left(\mathrm{K}_{0.38} \mathrm{Ba}_{0.81}\right) \mathrm{Ga}_{2} \mathrm{~S}_{4}$ & 2.03 & NS & $13.4 \times$ AGS & 1.064 & $10 \mathrm{~ns}$ & NS & $0.9 x$ AGS @ $2.1 \mu \mathrm{m}$ & 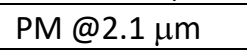 & NS & 51 \\
\hline $\mathrm{Cs}_{2} \mathrm{Ag}_{3} \mathrm{Sb}_{3} \mathrm{~S}_{7}$ & 2.02 & 3.53 & $2.3 \times$ AGS & 1.064 & $10 \mathrm{~ns}$ & NS & $0.6 x$ AGS @2.1 $\mu \mathrm{m}$ & PM @2.1 $\mu \mathrm{m}$ & NS & 45 \\
\hline \multirow[t]{2}{*}{$\mathrm{Sn}_{2} \mathrm{Ga}_{2} \mathrm{~S}_{5}$} & 2.02 & 20.1 & 9.7x AGS & 1.064 & $10 \mathrm{~ns}$ & NS & 1.6x AGS @2.1 $\mu \mathrm{m}$ & NPM @ $2.1 \mu \mathrm{m}$ & NS & 52 \\
\hline & 2.14 & 9.9 & 6.6x AGS & 1.064 & $10 \mathrm{~ns}$ & NS & $2.5 x @ 2.05 \mu \mathrm{m}$ & PM @2.05 $\mu \mathrm{m}$ & NS & 53 \\
\hline$\beta-\mathrm{SrCu}_{2} \mathrm{SnS}_{4}$ & 2.01 & NS & $2.3 \times$ AGS & 1.06 & $50 \mathrm{~ns}$ & NS & 1.1x AGS @2.09 $\mu \mathrm{m}$ & PM @2.09 $\mu \mathrm{m}$ & NS & 48 \\
\hline $\mathrm{NaGaIn}{ }_{2} \mathrm{Se}_{5}$ & 2.00 & 7.7 & $6.4 \times \mathrm{AGS}$ & 1.064 & $10 \mathrm{~ns}$ & 6.2 & 2.1 xAGS @1.91 $\mu \mathrm{m}$ & PM @1.91 $\mu \mathrm{m}$ & NS & 44 \\
\hline $\mathrm{Li}_{2} \mathrm{ZnSnSe}{ }_{4}$ & 2.0 & 300 & $\sim A G S$ & 1.064 & $30 \mathrm{ps}$ & 0.5 & $\chi^{(2)}=23 \mathrm{pm} / \mathrm{V}^{\mathrm{SL}}$ & $\mathrm{PM} \geq 2.5 \mu \mathrm{m}$ & $\mathrm{OQ}$ & 41 \\
\hline $\mathrm{PbGa}_{2} \mathrm{GeSe}_{6}$ & 1.96 & 5.61 & $3.7 \times$ AGS & 1.064 & $8 \mathrm{~ns}$ & 6.5 & $5 x$ AGS @2.05 ㅆm & PM @2.05 $\mu \mathrm{m}$ & NS & 54 \\
\hline $\mathrm{Cd}_{4} \mathrm{SiSe}_{6}$ & 1.95 & 15.5 & $7.1 \times$ AGS & 1.064 & $10 \mathrm{~ns}$ & NS & 1.9x AGS @2.05 $\mu \mathrm{m}$ & PM @2.05 $\mu \mathrm{m}$ & NS & 35 \\
\hline $\mathrm{Sr}_{6} \mathrm{Cu}_{4} \mathrm{Sn}_{4} \mathrm{~S}_{16}$ & 1.94 & NS & 1.0x AGS & 1.06 & NS & NS & 1.5x AGS @2.09 um & NPM & NS & 34 \\
\hline $\mathrm{Na}_{2} \ln _{4} \mathrm{SSe}_{6}$ & 1.92 & 6.9 & $5.8 \times$ AGS & 1.064 & $10 \mathrm{~ns}$ & 6.2 & 7x AGS @1.91 $\mu \mathrm{m}$ & NPM @1.91 $\mu \mathrm{m}$ & NS & 44 \\
\hline $\mathrm{RbInSn}_{2} \mathrm{Se}_{6}$ & 1.92 & 11.66 & 8.1x AGS & 1.064 & $8 \mathrm{~ns}$ & NS & 4.8x AGS @2.05 $\mu \mathrm{m}$ & PM @2.05 $\mu \mathrm{m}$ & NS & 55 \\
\hline $\mathrm{BaMnSnS}_{4}$ & 1.90 & 31.067 & 10x AGS & 1.064 & $10 \mathrm{~ns}$ & $0.3 \mathrm{~cm}^{2 *}$ & $1.2 x$ AGS @ $1.91 \mu \mathrm{m}$ & PM @ $1.9 \mu \mathrm{m}$ & NS & 38 \\
\hline Monoclinic $\mathrm{Ga}_{2} \mathrm{Se}_{3}$ & 1.86 & 29.6 & 12.6x AGS & 1.064 & $20 \mathrm{~ns}$ & NS & $\sim$ AGS @ $2.1 \mu \mathrm{m}$ & PM @ $2.1 \mu \mathrm{m}$ & NS & 56 \\
\hline$\gamma-\ln _{2} \mathrm{Se}_{3}$ & 1.84 & 17.1 & $7.3 \times$ AGS & 1.064 & $10 \mathrm{~ns}$ & NS & $2.6 x$ AGS @2.1 $1 \mu \mathrm{m}$ & PM @2.1 $\mu \mathrm{m}$ & NS & 57 \\
\hline $\mathrm{RbGaSn}_{2} \mathrm{Se}_{6}$ & 1.80 & 12.82 & 8.9x AGS & 1.064 & $8 \mathrm{~ns}$ & NS & 4.2x AGS @2.05 $\mu \mathrm{m}$ & PM @2.05 $\mu \mathrm{m}$ & NS & 55 \\
\hline $\mathrm{Ag}_{4} \mathrm{P}_{2} \mathrm{Se}_{6}$ & 1.76 & NS & $\sim A G S$ & 1.064 & $10 \mathrm{~ns}$ & NS & $0.5 x$ AGS @2.05 رm & PM @2.05 $\mu \mathrm{m}$ & NS & 58 \\
\hline $\mathrm{Ba}_{6} \mathrm{Cu}_{2} \mathrm{FeGe}_{4} \mathrm{~S}_{16}$ & 1.72 & NS & 2x AGSe & 1.064 & NS & NS & 15x AGSe @ $2.09 \mu \mathrm{m}$ & NPM & NS & 59 \\
\hline $\mathrm{SrCdSnSe}_{4}$ & 1.54 & 15.279 & $5 \times$ AGS & 1.064 & $10 \mathrm{~ns}$ & $0.196 \mathrm{~cm}^{2^{*}}$ & 1.5x AGS @2.04 $\mu \mathrm{m}$ & PM @2.04 $\mu \mathrm{m}$ & NS & 49 \\
\hline $\mathrm{Cu}_{2} \mathrm{CdSnS}_{4}$ & 0.92 & 200 & (0.67xAGS) & 1.064 & $30 \mathrm{ps}$ & 0.5 & $\chi^{(2)}=27 \mathrm{pm} / \mathrm{v}^{\mathrm{SL}}$ & $\mathrm{PM} \geq 2.1 \mu \mathrm{m}$ & $\mathrm{OQ}$ & 20 \\
\hline
\end{tabular}

$\mathrm{NS}=$ Not specified; $\mathrm{NM}=$ Not measured; $\mathrm{SC}=$ "high-quality $\mathrm{AGaS}_{2}$ crystal"; $\mathrm{OQ}=$ optical quality, i.e. obtained from commercial NLO crystal supplier.

$X=$ single crystal supplied from Anhui Institute of Optics and Fine Mechanics; ${ }^{\sharp G i v e n ~ i n ~ u n i t s ~ o f ~} \mathrm{~J} / \mathrm{cm}^{2}$

"spot area; ${ }^{* *}$ Authors note the differences in AGS reference: "Note that the measured LIDT of AgGaS2 in this work is larger than that in our previous work, which is induced by the different measured samples (single crystals in this work vs polycrystalline samples in our previous work)."

SLDetermined at the static limit.; \%For smallest particle size only 


\section{References}

(1) Su, Y.; Chen, Q.; Zhang, C.; Wang, R.; Wu, H.; Zhang, X.; Xing, G.; Yu, W. W.; Wang, X.; Zhang, Y.; Xiao, M. Two-photon-pumped perovskite semiconductor nanocrystal lasers. J. Am. Chem. Soc. 2016, 138, 3761-3768.

(2) Liu, B.-W.; Jiang, X.-M.; Li, B.-X.; Zeng, H.-Y.; Guo, G.-C. [Li[ $\left[\mathrm{LiCs}_{2} \mathrm{Cl}\right]\left[\mathrm{Ga}_{3} \mathrm{~S}_{6}\right]$ : A nanoporous framework of $\mathrm{GaS}_{4}$ tetrahedra with excellent nonlinear optical performance. Angew. Chem. Int. Ed. 2020, 59, 4876-4859.

(3) Zhang, J.-H.; Clark, D. J.; Brant, J. A.; Rosmus, K. A.; Jang, J. I.; Aitken, J. A., a-Li2 ZnGeS 4 : A wide bandgap diamond-like semiconductor with excellent balance between laser-induced damage threshold and second harmonic generation response. Chem. Mater. submitted.

(4) Yang, Y.; $\mathrm{Wu}, \mathrm{K}$.; $\mathrm{Wu}, \mathrm{X}$.; Zhang, B.; Gao, L. A new family of quaternary thiosilicates $\mathrm{SrA}_{2} \mathrm{SiS}_{4}(\mathrm{~A}=\mathrm{Li}, \mathrm{Na}, \mathrm{Cu})$ as promising infrared nonlinear optical materials. J. Mater. Chem. C 2020, 8 1762-1767.

(5) Li, G.; Chu, Y.; Zhou, Z. From AgGaS 2 to $\mathrm{Li}_{2} \mathrm{ZnSiS}_{4}$ : Realizing impressive high laser damage thresholds together with large second-harmonic generation response. Chem. Mater. 2018, 30, 602-606.

(6) Yang, L.-Q.; Jiang, X.-M.; Guo, G.-C. LiGa $0.54 \mathrm{In}_{0.46} \mathrm{~S}_{2}$ : A new infrared nonlinear optical material with large laser damage threshold designed by gallium substitution in LilnS 2. Inorg. Chem. Commun. 2020, 115, 107852.

(7) Li, G.-M.; Chu, Y.; Li, J.; Zhou, Z.-X. $\mathrm{Li}_{2} \mathrm{CdSiS}_{4}$, a promising IR NLO material with a balanced $E_{\mathrm{g}}$ and $\mathrm{SHG}$ response originating from the effect of $C d$ with $d^{10}$ configuration. Dalton Trans. 2020, 49, 1975-1980.

(8) Wu, K.; Yang, Z.; Pan, S. $\mathrm{Na}_{2} \mathrm{BaMQ}_{4}(\mathrm{M}=\mathrm{Ge}, \mathrm{Sn} ; \mathrm{Q}=\mathrm{S}$, Se): Infrared nonlinear optical materials with excellent performance and that undergo structural transformations. Angew Chem. Int. Ed. 2016, 55, 6713-6715.

(9) Wu, K.; Zhang, B.; Yang, Z.; Pan, S. New compressed chalcopyrite-like $\mathrm{Li}_{2} \mathrm{BaMIVQ}_{4}$ (MIV=Ge, Sn; Q=S, Se): Promising infrared nonlinear optical materials. J Am. Chem. Soc. 2017, 139, 414885-14888.

(10) Zhao, J.; Mei, D.; Yang, Y.; Cao, W.; Liu, C.; Wu, Y.; Lin, Z. $\mathrm{Rb}_{10} \mathrm{Zn}_{4} \mathrm{Sn}_{4} \mathrm{~S}_{17}$ : A chalcogenide with large laser damage threshold improved from the Mn-based analogue. Inorg. Chem. 2019, 58, 15029-15033.

(11) Lin, X.; Zhang, G.; Ye, N. Growth and Characterization of $\mathrm{BaGa}_{4} \mathrm{~S}_{7}$ : A New Crystal for Mid-IR Nonlinear Optics. Cryst. Growth Des. 2009, 9, 1186-1189.

(12) Li, Y.-Y.; Liu, P.-F.; Wu, L.-M. $\mathrm{Ba}_{6} \mathrm{Zn}_{7} \mathrm{Ga}_{2} \mathrm{~S}_{16}$ : A wide band gap sulfide with phase-matchable infrared NLO properties. Chem. Mater. 2017, 29, 5259-5266.

(13) Huang, Y.; Wu, K.; Cheng, J.; Chu, Y.; Yang, Z.; Pan, S. Li ZnGeS 4 : a promising diamond-like infrared nonlinear optical material with high laser damage threshold and outstanding second-harmonic generation response. Dalton Trans. 2019, 48, 4484-4488.

(14) Abudurusuli, A.; Li, J.; Tong, T.; Yang, Z.; Pan, S. LiBa ${ }_{4} \mathrm{Ga}_{5} \mathrm{Q}_{12}(\mathrm{Q}=\mathrm{S}$, Se): Noncentrosymmetric metal chalcogenides with a cesium chloride topological structure displaying a remarkable laser damage threshold. Inorg. Chem. 2020, 59, 5674-5682.

(15) Yang, L.-Q.; Ye, R.; Jiang, X.-M.; Liu, B.-W.; Zeng, H.-Y; Guo, G.-C. Ba $a_{13} \ln _{12} Z_{n_{7}} S_{38}$ and $B a_{12} I_{12} Z n_{8} S_{38}$ : infrared nonlinear optical chalcogenides designed by zinc-induced non-centrosymmetric transformation. J. Mater. Chem. C 2020, 8, 3688-3693.

(16) Liu, B.-W.; Jiang, X.-M.; Zeng, H.-Y.; Guo, G.C. $\left[\mathrm{ABa}_{2} \mathrm{Cl}\right]\left[\mathrm{Ga}_{4} \mathrm{~S}_{8}\right](\mathrm{A}=\mathrm{Rb}, \mathrm{Cs})$ : Wide-spectrum nonlinear optical materials obtained by polycation-substitution-induced nonlinear optical (NLO)-Functional motif ordering. J. Am. Chem. Soc. 2020, 142, 10641-10645.

(17) Hu, Q.; Ruan, K.; Wang, Y.; Ding, K.; Xu, Y. Synthesis and nonlinear optical properties of new gallium thiophosphate $\mathrm{Rb}_{2} \mathrm{Ga}_{2} \mathrm{P}_{2} \mathrm{~S}_{9}$. New J. Chem. 2019, 43, 12468-12474.

(18) Li, G.; Wu, K.; Liu, Q.; Yang, Z.; Pan, S. $\mathrm{Na}_{2} \mathrm{ZnG}_{2} \mathrm{~S}_{6}$ : A new infrared nonlinear optical material with good balance between large second-harmonic generation and high laser damage threshold. J. Am. Chem. Soc. 2016, 138, 7422-7428.

(19) Brant, J. A.; Clark, D. J.; Kim, Y. S.; Jang, J. I. Zhang, J.-H.; Aitken, J. A. Li ${ }_{2} \mathrm{CdGeS}_{4}$, A Diamond-Like semiconductor with strong second-order optical nonlinearity in the infrared and exceptional laser damage threshold. Chem. Mater. 2014, 26, 2045-2048.

(20) Rosmus, K. A.; Brant, J. A.; Wisneski, S. D.; Clark, D. J.; Kim, Y. S.; Jang, J. I.; Brunetta, C. D.; Zhang, J.-H.; Srnec, M. N.; Aitken, J. A. Optical nonlinearity in $\mathrm{Cu}_{2} \mathrm{CdSnS}_{4}$ and a/b- $\mathrm{Cu}_{2} \mathrm{ZnSiS}_{4}$ : Diamond-like semiconductors with high laser-damage thresholds. Inorg. Chem. 2014, 53, 7809-7811. 
(21) Li, S.-F.; Jiang, X.-M.; Liu, B.-W.; Yan, D.; Zeng, H.-Y.; Guo, G.-C. Strong infrared nonlinear optical efficiency and high laser damage threshold realized in quaternary alkali metal sulfides $\mathrm{Na}_{2} \mathrm{Ga}_{2} \mathrm{MS}_{6}(\mathrm{M}=\mathrm{Ge}, \mathrm{Sn})$ containing mixed nonlinear optically active motifs. Inorg. Chem. 2018, 57, 6783-6786.

(22) Luo, Z.-Z.; Lin, C.-S.; Cui, H.-H.; Zhang, W.-L.; Zhang, H.; He, Z.-Z.; Cheng, W.-D., "SHG Materials SnGa $\mathrm{Q}_{7}$ (Q=S, Se) Appearing with Large Conversion Efficiencies, High Damage Thresholds, and Wide Transparencies in the Mid-Infrared Region", Chem. Mater. 2014, 26, 2743-2749.

(23) Brant, J. A.; Clark, D. J.; Kim, Y. S.; Jang, J. I.; Weiland, A.; Aitken, J. A. Outstanding laser damage threshold in $\mathrm{Li}_{2} \mathrm{MnGeS}_{4}$ and tunable optical nonlinearity in diamond-like semiconductors. Inorg. Chem. 2015, 54, 28092819.

(24) Liu, C.; Mei, D. J.; Cao, W. Z.; Yang, Y.; Wu, Y. D.; Li, G. B.; Lin, Z. S. Mn-Based tin sulfide $\mathrm{Sr}_{3} \mathrm{MnSn}{ }_{2} \mathrm{~S}_{8}$ with a wide band gap and strong nonlinear optical response. J. Mater. Chem. C 2019, 7, 1146-1150.

(25) Liu, B.-W.; Zeng, H.-Y.; Zhang, M.-J.; Fan, Y.-H.; Cuo, G. C.; Huang, J.-S.; Dong, Z.-C. Syntheses, structures and nonlinear-optical properties of metal sulfides $\mathrm{Ba}_{2} \mathrm{Ga}_{8} \mathrm{MS}_{16}$ (M= Si, Ge). Inorg. Chem. 2015, 54, 976-981.

(26) Li, Y.-Y.; Wang, H.; Sun, B.-W.; Ruan, Q.-Q.; Geng, Y.-L.; Liu, P.-F.; Wang, L.; Wu, L.-M. Ba ${ }_{10} Z n_{7} \mathrm{M}_{6} \mathrm{Q}_{26}$ : Two new mid-infrared nonlinear optical crystals with T2 supertetrahedron 3D framework. Cryst. Growth Des. 2019, 19, 1190-1197.

(27) Li, Z.; Zhang, S.; Huang, Z.; Zhao, L.-D.; Uykur, E.; Xing, W.; Lin, Z.; Yao, J.; Wu, Y. Molecular Construction from $\mathrm{AgGaS}_{2}$ to $\mathrm{CuZnPS}_{4}$ : Defect-induced second harmonic generation enhancement and cosubstitution-driven band gap enlargement. Chem. Mater. 2020, 32, 3288-3296.

(28) Pang, Y.; Zhou, A.; Lin, C.; Cheng, W.; Chai, G.-L. Ba ${ }_{10} \mathrm{In}_{6} \mathrm{Zn}_{7} \mathrm{~S}_{10} \mathrm{Se}_{16}$ and $\mathrm{Ba}_{10} \mathrm{In}_{6} \mathrm{Zn}_{7} \mathrm{Se}_{26}$ : Two new infrared nonlinear optical materials with T2 super tetrahedron. J. Alloy Compd. 2019, 797, 356-362.

(29) Duan, R.; Lin, H.; Wang, Y.; Zhou, Y.; Wu, L. Non-centrosymmetric sulfides $\mathrm{A}_{2} \mathrm{Ba}_{6} \mathrm{MnSn}_{4} \mathrm{~S}_{16}$ (A=Li, Ag): syntheses, structures and properties. Dalton Trans. 2020, 49, 5914-5920.

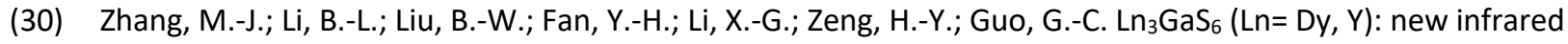
nonlinear optical materials with high laser induced damage thresholds. Dalton Trans. 2013, 42, 1422314229.

(31) Wu, K.; Yang, Z.; Pan, S. $\mathrm{Na}_{2} \mathrm{Hg}_{3} \mathrm{M}_{2} \mathrm{~S}_{8}$ (M=Si, Ge, and $\mathrm{Sn}$ ): New infrared nonlinear optical materials with strong second harmonic generation effects and high laser-damage thresholds. Chem. Mater. 2016, 28, 2795-2801.

(32) Wu, K.; Yang, Z.; Pan, S. $\mathrm{Na}_{4} \mathrm{MgM}_{2} \mathrm{Se}_{6}(\mathrm{M}=\mathrm{Si}$, Ge): The First Noncentrosymmetric Compounds with Special Ethane-like $\left[\mathrm{M}_{2} \mathrm{Se}_{6}\right]^{6-}$ Units Exhibiting Large Lase-Damage Thresholds. Inorg. Chem. 2015, 54, 10108-10110.

(33) Zhen, N.; Nian, L.; Li, G.; Wu, K.; Pan, S. A high laser damage threshold and good second-harmonic generation response in a new infrared NLO materials: $\mathrm{LiSm}_{3} \mathrm{SiS}_{7}$. Crystals 2016, 6, 121.

(34) Yang, Y.; Song, M.; Zhang, J.; Gao, L.; Wu, X.; Wu, K. Coordinated regulation on critical physicochemical performances activated from mixed tetrahedral anionic ligands in new series of $\mathrm{Sr}_{6} \mathrm{~A}_{4} \mathrm{M}_{4} \mathrm{~S}_{16}(\mathrm{~A}=\mathrm{Ag}, \mathrm{Cu}$; $\mathrm{M}=\mathrm{Ge}, \mathrm{Sn})$ nonlinear optical materials. Dalton Trans. 2020, 49, 3388-3392.

(35) Chen, J.; Lin, C.; Yang, S.; Jiang, X.; Shi, S.; Sun, Y; Li, B.; Fang, S.; Ye, N. Cd ${ }_{4} \mathrm{SiQ}_{6}(\mathrm{Q}=\mathrm{S}$, Se): Ternary infrared nonlinear optical materials with mixed functional building motifs. Cryst. Growth Des. 2020, 20, 2489-2496.

(36) Feng, J.-H.; Hu, C.-L.; Xu, X.; Li, B.-X.; Zhang, M.-J.; Mao, J.-G. AgGa $\mathrm{PS}_{6}$ : A new mid-infrared nonlinear optical material with a high laser damage threshold and a large second harmonic generation response.

(37) (a) Yao, J.; Mei, D.; Bai, L.; Lin, Z.; Yin, W.; Fu, P.; Wu, Y. BaGa $4 S_{7}$ : A new congruent-melting IR nonlinear optical material. Inorg. Chem. 2010, 49, 9212-9216. (b) Yao, J.; Yin, W.; Feng, K.; Li, X.; Mei, D.; Lu, Q.; Ni, Y.; Zhang, Z.; Hu, Z.; Wu, Y. Growth and characterization of $\mathrm{BaGa}_{4} \mathrm{Se}_{7}$ crystal. J. Cryst. Growth 2012, 346, 1-4.

(38) Lin, Y.-J.; Ye, R.; Yang, L.-Q.; Jiang, X.-M.; Liu, B.-W.; Zeng, H.-Y.; Guo, G.-C. BaMnSnS 4 and BaCdGeS4: infrared nonlinear optical sulfides containing highly distorted motifs with centers of moderate electronegativity. Inorg. Chem. Front. 2019, 6, 2365-2368.

(39) Yi, M.-Y.; Li, B.-X.; Lin, H.; Shi, Y.-F.; Ma, Z.; Wu, L.-M.; Wu, X.-T.; Zhu, Q.-L. Ternary mixed-metal Cd 4 GeS6: Remarkable nonlinear-optical properties based on a tetrahedral-stacking framework. Inorg. Chem. 2018, 57, 8730-8734.

(40) Zhang, J.-H.; Clark, D. J.; Weiland, A.; Stoyko, S. S.; Kim, Y. S.; Jang, J. I.; Aitken, J. A. $\mathrm{Li}_{2} \mathrm{CdGeSe}_{4}$ and $\mathrm{Li}_{2} \mathrm{CdSnSe}_{4}$ : biaxial nonlinear optical materials with strong infrared second-order responses and laserinduced damage thresholds influenced by photoluminescence. Inorg. Chem. Frontier 2017, 4, 1472-1484.

(41) (a) Zhang, J.-H.; Clark, D. J.; Brant, J. A.; Sinagra, C. W.; Kim, Y. S.; Jang, J. I.; Aitken, J. A. Infrared Nonlinear Optical Properties of Lithium-containing Diamond-like Semiconductors $\mathrm{Li}_{2} \mathrm{ZnGeSe}_{4}$ and $\mathrm{Li}_{2} \mathrm{ZnSnSe}{ }_{4}$. Dalton 
Trans. 2015, 44, 11212-11222. (b) Weiland, A.; Zhang, J.-H.; Clark, D. J.; Brant, J. A.; Sinagra, C. W. III; Kim, Y. S.; Jang, J. I.; Aitken, J. A. Correction: Infrared Nonlinear Optical Properties of Lithium-Containing Diamondlike Semiconductors $\mathrm{Li}_{2} \mathrm{ZnGeSe}{ }_{4}$ and $\mathrm{Li}_{2} \mathrm{ZnSnSe}$. Dalton Trans. 2017, 46, 10102-10104.

(42) Li, R.-A.; Zhou, Z.; Lian, Y.-K.; Jia, F.; Jiang, X.; Tang, M.-C.; Wu, L.-M.; Sun, J.; Chen, L. A2SnS 5 : A structural incommensurate modulation exhibiting strong second-harmonic generation and high laser-induced damage threshold (A=Ba, Sr). Angew Chem. Int. Ed. 2020, 59, 11861-11865.

(43) Zhou, A.; Lin, C.; Li, B.; Cheng, W.; Guo, Z.; Hou, Z.; Yuan, F.; Chai, G.-L. Ba $\ln _{6} Z_{4} \mathrm{n}_{4} \mathrm{Se}_{19}$ : A high performance infrared nonlinear optical crystal with $\left[\mathrm{InSe}_{3}\right]^{3-}$ trigonal planar functional motifs. J. Mater. Chem. C 2020, 8, 7947-7955.

(44) Li, S.-F.; Jiang, X.-M.; Liu, B.-W.; Yan, D.; Lin, C.-S.; Zeng, H.-Y.; Guo, G.-C., Superpolyhedron-Built Second Harmonic Generation Materials Exhibit Large Mid-Infrared Conversion Efficiencies and High Laser-Induced Damage Thresholds. Chem. Mater. 2017, 29, 1796-1804.

(45) Yang, G.; Li, L.-H.; Wu, C.; Humphrey, M. G.; Zhang, C. lonothermal synthesis of metal chalcogenides $\mathrm{M}_{2} \mathrm{Ag}_{3} \mathrm{Sb}_{3} \mathrm{~S}_{7}(\mathrm{M}=\mathrm{Rb}, \mathrm{Cs})$ displaying nonlinear optical activity in the infrared region. Inorg. Chem. 2019, 58, $12582-15589$.

(46) Guo, S.-P.; Chen, X.; Sun, Z.-D.; Chi, Y.; Liu, B.-W.; Jiang, X.-M.; Li, S.-F.; Xue, H.-G.; Deng, S.; Duppel, V.; Köhler, J.; Guo, G.-C. Large second harmonic generation (SHG) effect and high laser-induced damage threshold (LIDT) observed coexisting in gallium selenide. Angew. Chem. Int. Ed. 2019, 58, 8087-8091.

(47) Li, X.-H.; Chen, Y.-T.; Xue, H.; Guo, S.-P. KInSi ${ }_{1.32} \mathrm{Sn}_{0.68} \mathrm{Se}_{6}$ : An infrared nonlinear optical material containing three types of tetrahedral units. Inorg. Chem. 2020, 59, 5823-5827.

(48) Yang, Y.; Wu, K.; Zhang, B.; Wu, X.; Lee, M.-H. Infrared nonlinear optical polymorphs $\alpha$-and $\beta-\mathrm{SrCu}_{2} \mathrm{SnS}_{4}$ exhibiting large second harmonic generation responses with requite phase-matching behavior. Chem. Mater. 2020, 32, 1281-1287.

(49) Lin, Y.-J.; Liu, B.-W.; Ye, R.; Jiang, X.-M.; Yang, L.-Q.; Zeng, H.-Y.; Guo, G.-C. SrCdSnQ $(Q=S$, Se): Infrared nonlinear optical chalcogenides with mixed NLO-active and synergetic distorted motifs. J. Mater. Chem $C$ 2019, 7, 4459-4465.

(50) Li, M.-Y.; Ma, Z.; Li, B.; Wu, X.-T.; Lin, H.; Zhu, Q.-L. HgCuPS 4 : An exceptional infrared nonlinear optical material with defect diamond-like structure. Chem. Mater. 2020, 32, 4331-4339.

(51) Li, Y.-N.; Chi, Y.; Sun, Z.-D.; Xue, H.; Suen, N.-T.; Guo, S.-P. Partial substitution induced centrosymmetric to noncentrosymmetric structure transformation and promising second-order nonlinear optical properties of $\left(\mathrm{K}_{0.38} \mathrm{Ba}_{0.81}\right) \mathrm{Ga}_{2} \mathrm{Se}_{4}$. Chem. Commun. 2019, 55, 13701-13704.

(52) Shi, Z.-H.; Chi, Y.; Sun, Z.-D.; Liu, W.; Guo, S.-P. $\mathrm{Sn}_{2} \mathrm{Ga}_{2} \mathrm{~S}_{5}$ : A type of IR nonlinear-optical material. Inorg. Chem. 2019, 58, 12002-12006.

(53) Li, M.-Y.; Li, B.; Lin, H.; Ma, Z.; Wu, L.-M.; Wu, X.-T.; Zhu, Q.-L. $\mathrm{Sn}_{2} \mathrm{Ga}_{2} \mathrm{~S}_{5}$ : A polar semiconductor with exceptional infrared nonlinear optical properties originating from the combined effect of mixed asymmetric building motifs. Chem. Mater. 2019, 31, 6268-6275.

(54) Luo, Z.-Z.; Lin, C.-S.; Cui, H.-H.; Zhang, W.-L.; Zhang, H.; Chen, H.; He, Z.-Z.; Cheng, W.-D. PbGa ${ }_{2} M S e_{6}$ (M = Si, Ge): Two Exceptional Infrared Nonlinear Optical Crystals. Chem. Mater. 2015, 27, 914-922.

(55) Lin, H.; Chen, H.; Zheng, Y.-J.; Yu, J.-S.; Wu, X.-T.; Wu, L.-M. Two excellent phase-matchable infrared nonlinear optical materials based on 3D diamond-like frameworks: $\mathrm{RbGaSn}_{2} \mathrm{Se}_{6}$ and $\mathrm{RbInSn}_{2} \mathrm{Se}_{6}$. Dalton Trans. 2017, 46, 7714-7721.

(56) Xi, Q.-T.; Sun, Z.-D.; Chi, Y.; Xue, H.-G.; Guo, S.-P. Monoclinic gallium selenide: an AgGaS 2 -type structure variant with balanced infrared nonlinear optical performance. J. Mater. Chem. C 2019, 7, 11752-11756.

(57) Chi, Y.; Sun, Z.-D.; Xu, Q.-T.; Xue, H.-G.; Guo, S.-P. Hexagonal $\ln _{2} \mathrm{Se}_{3}$ : A defect wurtzite-type infrared nonlinear optical material with moderate birefringence contribute by unique InSe $\mathrm{s}_{5}$ unit. ACS Appl. Mater. Interfaces 2020, 12, 17699-17705.

(58) Xia, H.-P.; Ma, Q. Experimental study on nonlinear-optical properties of $\mathrm{Ag}_{4} \mathrm{P}_{2} \mathrm{Se}_{6}$. J. Alloy Compd. 2019, 780, 727-733.

(59) Cao, W.; Mei, D.; Yang, Y.; Wu, Y.; Zhang, L.; Wu, Y.; He, X.; Lin, Z.; Huang, F. From $\mathrm{CuFeS}_{2}$ to $\mathrm{Ba}_{6} \mathrm{Cu}_{2} \mathrm{FeGe}_{4} \mathrm{~S}_{16}$ : rational band gap engineering achieves large second-harmonic generation together with high laser damage threshold. Chem. Commun. 2019, 55, 14510-14513. 